\title{
Hybrid Rossby-shelf modes in a laboratory
}

\section{ocean}

\author{
Onno BoKhove *AND Vijaya Ambati
}

Department of Applied Mathematics, Univ. of Twente, Enschede, The Netherlands

${ }^{*}$ Corresponding author address: Onno Bokhove, Department of Applied Mathematics - Institute of Mechanics, Processes, and Control, Twente- University of Twente, Enschede, P.O. Box 217, The Netherlands. E-mail: o.bokhove@math.utwente.nl 


\begin{abstract}
Idealized laboratory experiments reveal the existence of forced dissipative hybrid Rossby shelf modes. The laboratory ocean consists of a deeper ocean, accommodating basin scale Rossby modes, and a coastal step shelf, accommodating trapped shelf modes. Planetary Rossby modes are mimicked in the laboratory via a uniform topographic slope in the North-South direction. Hybrid modes are found as linear modes in numerical calculations and similar streamfunction patterns exist in streak photography of the rotating tank experiments. These numerical calculations are based on depth-averaged potential vorticity dynamics with Ekman forcing and damping. Preliminary nonlinear calculations explore the deficiencies observed between reality and the linear solutions. The aim of the work is twofold: to show that idealized hybrid Rossby shelf modes exist in laboratory experiments, and to contribute in a general sense to the discussion on the coupling and energy exchange associated with hybrid modes between shallow coastal seas and deep ocean basins.
\end{abstract}




\section{Introduction}

There is evidence that planetary scale Rossby waves have been generated off the western coast of the U.S.A. either by unstable coastal boundary currents, or by coastal waves matching in frequency and scale, cf. Kelly et al. (1998). Bokhove and Johnson (1999) therefore investigated the matching of planetary Rossby modes with coastal shelf modes in a cylindrical basin. Otherwise said, linear free modes were calculated with so-called semi-analytical "mode matching" techniques, as well as linear forced dissipative finite element methods, to find resonances. Two parameter regimes were considered: an ocean one and a laboratory analogue. These laboratory scale hybrid Rossby shelf modes had been considered with validating laboratory rotating tank experiments in mind.

Such an experimental validation is the topic of the present paper. Planetary barotropic Rossby modes have been shown before in the laboratory using the analogy between planetary $\beta$-plane Rossby modes and topographic shelf modes for a uniform basin scale North-South background topography, for example in the classic book of Greenspan (1968). Rotating tank experiments geared towards enforcing resonant hybrid coastal and planetary modes appear to be (relatively) new. In preparation of the rotating tank experiments, linear forced-dissipative finite element calculations of barotropic potential vorticity dynamics have revealed the resonant frequencies of two primary hybrid Rossby shelf modes. These primary forcing frequencies were then used to drive the harmonic "wind" forcing provided via the Ekman pumping and suction due to an oscillating rigid lid. Various forcing strengths have been imposed, in which a match with the linear calculations is best suited by weak forcing while better visualization requires a larger signal-to-noise ratio and consequently stronger forcing. The 
latter promotes, however, the emergence of nonlinear effects. We therefore also compare the experimental streamfunction fields with some nonlinear simulations of barotropic potential vorticity dynamics, in an attempt to explain the differences between linear theory and the experimental results under stronger forcing.

In OGCM's (ocean general circulation models) the coastal regions are often under resolved; furthermore it is hypothesized that significant energy exchange takes place between the deeper ocean and the shallower coastal zones, e.g., Wunsch (2004). Vertical walls placed in the shallow seas are generally used in coastal zones as lateral boundaries in OGCM's, while in reality mass, momentum and energy are transferred across these virtual vertical walls. As a consequence, such an exchange would not be resolved or modelled properly in the OGCM's. Suitable parameterizations of these under resolved processes would therefore be required in the coastal zones of OGCM's. The laboratory experiments and finite element calculations we present aim to serve as an idealized barotropic system to investigate this modal coupling between basin and coastal scale dynamics.

The outline is as follows. Barotropic potential vorticity dynamics is introduced in $\S 2$, and linear finite element calculations are presented to find the relevant forcing frequencies. These forcing frequencies are a building block in $\S 3$, where the experimental set-up and results are presented. Preliminary nonlinear simulations in $\S 4$ indicate the effects of strong forcing on the dynamics observed. A short conclusion is found in $\S 5$. 


\section{Rigid-lid potential vorticity model}

a. Nonlinear model

The forced-dissipative evolution of vertical vorticity $\omega$ in a rigid-lid model is governed by the following dimensional system of equations

$$
\begin{aligned}
\frac{\partial \omega}{\partial t}+J\left(\Psi, \frac{f+\omega}{H(\mathbf{x})}\right) & =\operatorname{curl} \mathbf{F}(\mathbf{x}, t)-\frac{(f+\omega)}{H(\mathbf{x})} \alpha D_{E} \omega \\
\omega & =\boldsymbol{\nabla} \cdot\left(\frac{1}{H(\mathbf{x})} \nabla \Psi\right)
\end{aligned}
$$

where the horizontal coordinates are $\mathbf{x}=(x, y)^{T}, t$ is time, $\boldsymbol{\nabla}=\left(\partial_{x}, \partial_{y}\right)^{T}=\left(\partial / \partial_{x}, \partial / \partial_{y}\right)^{T}$, the Jacobian $J(P, Q)=\partial_{x} P \partial_{y} Q-\partial_{y} P \partial_{x} Q$ for functions $P=P(x, y, t)$ and $Q=Q(x, y, t)$, the Coriolis parameter $f=f(x, y)$, the transport streamfunction is $\Psi$, total depth $H(\mathbf{x})$, forcing curl $\mathbf{F}$, and Ekman layer depth $D_{E}=\sqrt{\nu / \Omega_{r}}$ with (effective) viscosity $\nu$. For the laboratory case $f=f_{0}=2 \Omega_{r}$ is constant with $\Omega_{r}$ the rotation rate of the domain, while for the planetary case a $\beta$-plane approximation is used (e.g., Pedlosky (1987)) and $f=f_{0}+\beta y$ for constant $\beta$. The forcing either relates to the windstress or the differential velocity of the rigid lid, provided by Ekman suction or pumping, respectively (e.g., Pedlosky (1987, 1996)). Hereafter, unless otherwise indicated, we consider the laboratory case with

$$
\operatorname{curl} \mathbf{F}(\mathbf{x}, t)=\frac{(f+\omega)}{H} \frac{1}{2} D_{E} \omega_{T}
$$

in which the vorticity at the top rigid lid

$$
\omega_{T}=\partial_{x} v_{T}-\partial_{y} u_{T}
$$

is related to the velocity $\mathbf{v}_{T}(x, y, t)=\left(u_{T}, v_{T}\right)^{T}$ of the driven rigid lid. Ekman damping is assumed to be valid in the transition region between a quasigeostrophic deep ocean and 
the ageostrophic coastal zone, even though the topography is rapidly changing from open ocean to coastal zone. For the rigid-lid case $\alpha=1$; it results in twice the amount of Ekman damping relative to the case with a free surface for which $\alpha=1 / 2$. The above system can be derived using classical methods (Pedlosky 1987; Pedlosky et al. 2007).

The dimensional equations are scaled with radius $R$ of the cylindrical domain, a typical depth $H_{0}$ of the domain and the Coriolis parameter $f_{0}$ (in which starred variables are dimensional):

$$
\begin{aligned}
& t^{\star}=t / f_{0}, \quad\left(x^{\star}, y^{\star}\right)=R(x, y), \quad \Psi^{\star}=\Psi R^{2} H_{0} f_{0}, \\
& \omega^{\star}=f_{0} \omega, \quad H^{\star}=H_{0} H, \quad f^{\star}=f_{0} f .
\end{aligned}
$$

The potential vorticity of the fluid is defined by

$$
\xi=(f+\omega) / H
$$

The evolution of this potential vorticity weighted by the depth $H$ follows by scaling with (4) and rewriting the nondimensionalized form of the system (1) into

$$
\begin{aligned}
H \frac{\partial \xi}{\partial t}+\nabla \cdot(\mathbf{U} \xi) & =\kappa \xi\left(\frac{1}{2} \omega_{T}+\alpha f-\alpha H \xi\right) \approx \kappa\left(\frac{1}{2} \frac{\omega_{T}}{H}+\alpha \frac{f}{H}-\alpha \xi\right) \\
\mathbf{U} & =\nabla^{\perp} \Psi \\
\nabla \cdot\left(\frac{1}{H(\mathbf{x})} \nabla \Psi\right) & =H \xi-f
\end{aligned}
$$

with transport velocity $\mathbf{U}$, two-dimensional curl operator $\nabla^{\perp}=\left(-\partial_{y}, \partial_{x}\right)^{T}$, and parameter:

$$
\kappa=D_{E} / H_{0}=\sqrt{\nu / \Omega_{r}} / H_{0}
$$

A cylindrical domain $\Omega$ is considered with $\Psi=0$ at the boundary $\partial \Omega: r=R$; and initial conditions $\xi=\xi(x, y, 0)$; we also used that $\xi \approx 1 / H$ to simplify forcing and damping terms. 
The numerical (dis)continuous Galerkin finite element discretization used is based on formulation (6), by extending the inviscid formulation in Bernsen et al. (2006); it couples the hyperbolic potential vorticity equation (6a) to the elliptic equation (6c) for the transport streamfunction and is advantageous for complex-shaped domains. Instead of a classical continuous finite element method as used for the streamfunction, potential vorticity is descretized discontinuously. For smooth profiles of potential vorticity, the numerical discontinuities between elements are negligible and scale with the mesh size and order of accuracy. The numerical method conserves vorticity and energy for infinitesimally small time steps in the inviscid and unforced case, while enstrophy is slightly decaying for the upwind flux used. The weak formulation of this finite element method is given in Appendix A. The method provides an alternative to classical numerical methods and is well-suited for complex-shaped domains, mesh and order $(h$ - and $p$ - $)$ refinement.

\section{b. Linear model and hybrid Rossby-shelf modes}

We have performed laboratory experiments to assess whether hybrid Rossby-shelf modes exist that couple planetary scale Rossby modes with coastal scale shelf modes. In the laboratory experiments a uniformly rotating tank is used with rotation frequency $\Omega_{r}$. Consequently, there is no planetary variation of the background rotation in the North-South direction. We consider the Northern hemisphere and define a North-South direction indirectly by introducing a background slope $s=s(y)$ in the $y$-direction, which is related mathematically to the $\beta$ effect (e.g., Greenspan (1968)). Hence, the nondimensional $\beta$ parameter $\beta=s / \bar{H}$ at leading

order for a mean depth $\bar{H}$. (Dimensionally $\beta^{\star}=\beta f_{0} / R$ with $\beta^{\star} \approx \mathrm{d} f^{\star} / \mathrm{d} y^{\star}$ with starred 
dimensional variables and coordinates.) In addition, a step shelf topography is chosen with a sudden change in depth at $r=R_{s}<R$ from the mean deep ocean value $H_{2}$ to the mean shallow coastal one $H_{1}<H_{2}$. The topography in the laboratory domain therefore consists of an interior deep ocean on a topographic $\beta$-plane with slope $s_{2}=\beta H_{2}$, and likewise a shallow shelf ocean with $s_{1}=\beta H_{1}$. Hence, $H=H_{1}-s_{1} y$ for $r>R_{s}$ on the axisymmetric shelf and $H=H_{2}-s_{2} y$ in the deep ocean for $r<R_{s}$. A sketch of the domain has been given in Fig. 1.

To assess at which forcing frequencies we expect resonant responses, we numerically solved the linear counterpart of nonlinear dynamics (6). The flow regime of interest concerns quasi-steady state dynamics under harmonic forcing

$$
\omega_{T}=2 \sigma \Delta \theta e^{i \sigma t}
$$

with forcing frequency $\sigma$, angle $\Delta \theta$ and complex number $i^{2}=-1$. Due to this enforced harmonic behavior the linear dynamics can be reduced to a spatial problem. A secondorder Galerkin finite-element model (FEM) discretization in space was used with piecewise linear basis and test functions. In a first set of simulations we took 4671 nodes and 4590 quadrilateral elements on an unstructured mesh. In this FEM, a matrix system results of the form $A_{i j} \Psi_{j}=b_{i}$ with $\Psi_{j}$ the values on the node with index $j, b_{i}$ the result of the forcing, and matrix elements $A_{i j}$ the combination of the rotational advective and dissipative terms. To test the finite element implementation, we successfully recovered the forced-dissipative analogues of the free planetary Rossby modes and their frequencies (Appendix A.§1).

The forced-dissipative response of the laboratory ocean, described above and sketched in Fig. 1, is displayed in Fig. 2 for $\kappa=\sqrt{\nu / \Omega_{r}} / H_{0}=0.042$, and $\Delta \theta=2 \pi$ (using laboratory 
values of the viscosity of water $\left.\nu=10^{-6} \mathrm{~m}^{2} / \mathrm{s}, \Omega_{r}=2 \mathrm{~s}^{-1}, H_{0}=R^{\star}=0.17 \mathrm{~m}\right)$. The streamfunction field at maximum response is shown in Fig. 3 at forcing frequency $\sigma=$ 0.0613. When the experiments were performed in late 1997, O.B. calculated a resonance at $\sigma=0.0612$ for fewer and less-accurate triangular elements, and used that slightly different resonant frequency instead in the laboratory. This small difference, however, falls within the accuracy to which the forcing frequency could be determined in the laboratory. Another resonant frequency resides at $\sigma=0.0878$ (calculation FEM 2009), where O.B. found $\sigma=$ 0.0871 earlier (calculation FEM 1997) with streamfunction fields shown in Fig. 4. The modal structure for either value, $\sigma=0.0878$ (or 0.0871 ), is the same and the mode relates most clearly to the $m=2$ shelf mode. In these cases, the hybrid Rossby-shelf mode is a combination of an azimuthal mode number $m=0$-Rossby mode in the deep ocean absorbing into a $m=2$-shelf mode at the Western shelf, which after traversing counterclockwise along the Southern shelf edge at $R_{s}=0.8 R$ radiates again into the deep-ocean "planetary" Rossby mode. Such behavior is suggested directly from the dispersion relations (A6) and (A15) of the invisicid or free planetary Rossby wave and shelf modes, plotted separately in Fig. 5. The exact solution of the planetary Rossby mode is given in (A5) and (A6) and the one of the shelf mode in (A15). The modal structure of the free planetary Rossby mode clearly includes the East-West structure in its explicit $x$-dependence, while the free shelf mode is well described solely in terms of polar coordinates. The spatial structure of the hybrid-shelf mode, for the case with both $\beta$-plane topography and a step shelf, is thus seen to correspond best by a combination of the $m=0$ planetary free Rossby mode, with its explicit $x-$ and $r$-dependence, and the $m=2$ free shelf mode, with its explicit $r$ - and $\theta$-dependence. In contrast, the modal structures of the $m=1$ free Rossby mode and the $m=1$ free shelf mode 
don't match that well, have more structure and are therefore more affected by damping. The resonant peak expected at around $\sigma=0.04$ for this $m=1$-mode appears to be wiped out in Fig. 2. See also the relevant $m=1$ modes displayed in Figs. 3 and 4 in Bokhove and Johnson (1999).

In the nonlinear numerical simulations described in the next section, instead of the abrupt step shelf break a smoothed shelf break is introduced between $R_{s}-\epsilon<r<R_{s}+\epsilon$. The finite element mesh contains regular nodes placed at the circles with radii $R_{s} \pm \epsilon$ and $R$; then random nodes are added subject to a minimum distance criterion outside the shelf break; subsequent triangulation yields a triangular mesh and additional nodes are placed at the centroid and midpoints of the edges of each triangle to further divide the triangular mesh into a quadrilateral one. The shelf break contains two elements across and upon mesh refinement the value of $\epsilon$ decreases and hence the shelf break also becomes more narrow. For such smoothed topography, the linear forced-dissipative response and the streamfunction field at the maximum resonance are given in Fig. 6 and Fig. 7 for $\epsilon=0.0314$. Relative to the abrupt shelf topography with resonant forcing frequency $\sigma=0.0613$, the resonant frequency in the new calculation of the linearized system has lowered by $6 \%$ to $\sigma=0.0577$ while the actual fields at resonance remain highly similar. 


\section{Laboratory experiments}

a. Experimental set-up

The laboratory tank had the following dimensions: $R^{*}=16.7 \mathrm{~cm}, R_{s}=0.8 R$, and $\beta=\beta^{*} R^{*} /\left(2 \Omega_{r}\right)=s_{i} / H_{i}=0.3125$ for $i=1,2$. The topography was cut out of foam, subsequently made smooth with paste, and fit tightly into the cylindrical tank. The average shelf and interior depths were $H_{1}=0.6 R$, and $H_{2}=0.8 R$, respectively. The corresponding laboratory set-up is sketched in Fig. 1. Dimensionless variables therein are defined in terms of the dimensional tank radius $R^{\star}$ and $H_{0}=R^{\star}$. The glass plate on top of the water column was harmonically oscillating in a horizontal plane, its motion driven by a programmable stepping motor connected to the plate with a driving belt. The non-dimensional azimuthal velocity of the rigid lid is

$$
v_{T}=r \sigma \Delta \theta \cos \sigma t
$$

cf. (8), with $\Delta \theta$ the maximum angle of the lid reached over the forcing period $T=2 \pi / \sigma$. A thin horizontal light sheet had been constructed with a Tungsten lamp including a linear filament and a lens. The black background permitted optimal reflection of light from whitish pliolite particles of diameter $150-250 \mu \mathrm{m}$, suspended in the flow. An analogue photo camera mounted above the rotating tank tracked the movement of the particles. The whole configuration was placed on a uniformly rotating table with $\Omega=1 \mathrm{~s}^{-1}$. An approximate steadily oscillating state was achieved by spinning up the table for about 30min. (i.e., $30-50$ forcing periods). 


\section{b. Coupled modes}

Numerous experiments were carried out for a few forcing frequencies. For each experiment, streak photography was obtained with 2 to 4 s exposure time. The main forcing frequencies used in the laboratory experiments were calculated with a finite element model of the linearized equations, as explained in $§ 2 \mathrm{~b}$. We report here solely four sets of experiments, deemed best regarding their visual resolution and the hybrid character of the Rossby-shelf mode.

The two sets of eight images in Fig. 9 and Fig. 10 give an impression of the flow during one forcing period of $51.3 \mathrm{~s}$, i.e., with dimensional frequency $\sigma^{\star}=0.1226 \mathrm{~s}^{-1}(\sigma=0.0612)$. It nearly corresponds to a numerically calculated forced-dissipative resonance for a hybrid Rossby-shelf mode of the rigid-lid model (6) linearized around a state of rest, see Fig. 2 and Fig. 3. The underlying Rossby mode has azimuthal mode number zero, while the underlying shelf mode has azimuthal number $m=1,2$. The shelf break is visible as a thin whitish line at $r=0.8 R$. In the time sequence from top left to bottom right, a Rossbymode circulation cell in the deep interior "ocean" travels westward where it absorbs onto the shelf and propagates counterclockwise (in the Northern Hemisphere) as a trapped shelf mode circulation cell. On the Eastern boundary this shelf mode radiates into a planetary Rossby mode. Apart from the striking qualitative resemblance with linear forced-dissipative calculations in Fig. 3, discrepancies occur in the North-West, presumably due to nonlinear effects, and in the East where the shelf mode disappears, presumably due to strong damping. The rigid lid or glass plate has rotated from zero to a relatively large angles $2 \pi$ and $\pi$ (Fig. 10 and Fig. 9, respectively) and back during a period. The "nonlinear" oscillations in the North- 
West corner at $t=14,42 \mathrm{~s}$ in Fig. 10 are larger under the greater forcing amplitude than at $t=0,28 \mathrm{~s}$ in Fig. 9. These oscillations diminish even more when the forcing amplitude is reduced to $\pi / 2$, which is not shown here. The experimental dilemma is that a comparision with linearized modal solutions requires a weak forcing while good visualisation requires strong forcing for the streak photography used. Particle Image Velocimetry techniques could have been used for weak forcing as well, but were not avialable in 1997 at the rotating tank facilities in Woods Hole. Although the tank dimensions are not shallow, the simplifying assumption has been that rotation is sufficiently strong to render the flow to be nearly two-dimensional outside the thin Ekman top and bottom boundary layers, and the sidewall boundary layers as well as the internal and boundary layers at the shelf break.

To compare the amplitudes observed and calculated, the streaks under 4s exposure are compared with streak lengths in the calculation for $\sigma=0.00613$ and $\Delta \theta=\pi$ in Fig. 11 . Note that the calculated streaks are weaker, about $40 \%$, than the observed ones. Such a difference also occurs for forcing with $\Delta \theta=2 \pi$ in Fig. 12. The precise location of the mode around resonance, and hence its amplitude, as well as nonlinear shifts, might cause this discrepancy. The dimensionless speed at $(x, y)=(-0.11,0.11)$ is about 0.0276 and at the Southern shelf the maximum speed is about 0.0773 in Fig. 11(a). The speed at $(x, y)=(0,0)$ is about 0.0543 and at the Southern shelf the maximum speed is about 0.1087 in Fig. 12(a).

Similarly, the linear forced disspative mode calculated at $\sigma=0.0878$ corresponds reasonably well with the observed mode for $\sigma=0.0871$ (based on the FEM calculation in 1997) in Fig. 13 and Fig. 14, which are the observations under stronger and weaker forcing $\Delta \theta=2 \pi, \pi / 2$

Finally, we conclude that hybrid-Rossby shelf modes exist and can be succesfully visu- 
alized and measured in the laboratory; they correspond well with linear forced-dissipative calculations at a similar frequency. Discrepancies between the experimental and numerical flow patterns are observed especially at the North-Western boundary. Further nonlinear simulations aim to explain this discrepancy.

\section{Laboratory results versus numerical simulations}

Nonlinear simulations at resonance frequency $\sigma=0.0613$ have been performed, starting from a state of rest and with sinusoidal forcing. The forcing period is thus 102.5 time units; in addition $\kappa=0.0042$, with a smoothed shelf break of width $2 \epsilon=0.0628$, and $\Delta \theta=2 \pi$. The value of $\kappa=0.0042$ implies that start-up transients disappear below $1 \%$ of their initial value within about 11 periods, cf. the energy and enstrophy graphs versus time in Fig. 15. We note that the solution appears quasi-periodic for $t>800$. Shown is the solution over period 20 in Fig. 16 (i.e., from $t=1947.5$ to 2050.0). A second-order spatial and third-order temporal discretization has been used; single and double-resolution runs have been performed with 4671 and 10363 nodes, and 4590 and 10242 elements; the former are shown but agree well with the latter. These nonlinear simulations reveal the cause of the disturbances in the North-West corner of the domain at $t=14,42 \mathrm{~s}$ in Fig. 10: a vortex starts to roll up on the Northern shelf once the cell of the Southern shelf mode starts to radiate into a basin Rossby mode; subsequently the vortex gets advected counterclockwise around the domain by the basin Rossby mode, and is dissipated once the new forcing cycle starts, see Fig. 16 and Fig. 17 in tandem. The simulated potential vorticity field displayed is less smooth than the streamfunction fields and displays more structure, including a vortex shedding. 


\section{Conclusion}

Hybrid Rossby shelf modes were shown to exists analytically and numerically before in Bokhove and Johnson (1999). Based on depth-averaged potential vorticity dynamics, we showed numerically that these hybrid modes also emerged as linear forced dissipative solutions on a laboratory scale. These hybrid modes matched the largest planetary scale Rossby mode to a trapped shelf mode, the latter propagating around the Southern shelf. The calculated frequencies of the two dominant hybrid modes were used as driving frequency in laboratory tank experiments, based on the linearized calculations. Therein a driven lid provided the Ekman forcing. The spatial structure of the streamfunction fields in the linear calculations and the observed streamfunction fields in streak photography agreed well or reasonably well in the weaker and stronger forcing cases. Discrepancies in amplitude and structure were attributed to nonlinear effects, and nonlinear simulations of the depth-averaged flow suggested observed differences to be due to a vortex generated and shed off the Northern shelf by the large Westward propagating planetary Rossby mode in the deep basin. The minimum and maximum amplitudes in the calculations of the linear and nonlinear model differed; in the former we observed values of -0.005 and 0.009 and in the latter values of -0.014 and 0.005. The simulations provided extra information on potential vorticity dynamics which was unavailable from the laboratory observations.

Even though the topography used is still simple, the exhibited mode merging shows that

the linear normal modes rapidly obtain a complicated structure. The distinction between trapped shelf modes and planetary modes becomes less clear in complex domains, and is a bit artificial as both modes emerge from the spatial structure in the background potential 
vorticity. Vortical normal modes have recently been used to explain temporal variability in the Mascarene Basin (Weijer 2008), and Norwegian and Greenland Gyres (LaCasce et al. 2008). Unexplored yet interesting aspects in the idealized ocean basin used by us concern the effects of a mid-ocean ridge (cf. Pedlosky (1996)) on the communication between two separate deep ocean half basins connected only via coastal shelfs, and numerical parameterization of under resolved shelf mode dynamics on the deep ocean dynamics as a way to explore energy exchange through an effective, permeable boundary.

\section{Acknowledgments.}

It is a great pleasure to acknowledge the assistance of John Salzig in the laboratory. Without his help the experiments would have failed. The laboratory experiments were performed while O.B. was a postdoctoral scholar at the Woods Hole Oceanographic Institution (1996-1997); preliminary results were posted in Bokhove (1999). The encouragements and advice of Karl Helfrich, Joe Pedlosky, and Jack Whitehead have, as always, been of great value. Jack Whitehead also kindly allowed O.B. to use the rotating table in his laboratory, and additional support came via Joe Pedlosky's NSF-grant OCE-9901654, in 1997. 


\section{APPENDIX A}

\section{(Dis)continuous Galerkin finite element discretization}

a. Weak formulation

A (dis)continuous Galerkin finite element method is used to discretize the following generalized system of equations

$$
\begin{aligned}
\frac{1}{A} \frac{\partial \xi}{\partial t}+\nabla \cdot(\mathbf{U} \xi) & =\kappa\left(\frac{1}{2} A \omega_{T}+\alpha A D-\alpha \xi\right), \\
\mathbf{U} & =\nabla^{\perp} \Psi, \\
\nabla \cdot(A \nabla \Psi)-B \Psi & =\xi / A-D,
\end{aligned}
$$

with $A=A(x, y)>0, B=B(x, y) \geq 0$ and $D=D(x, y)$, and for a scaling in which $D \approx D_{0}=1$ in a relevant leading order way. Here, only a singly-connected domain $\Omega$ is considered with boundary $\partial \Omega$; it is cylindrical with radius $R$ and $\Psi=0$ at $\partial \Omega: r=R$. In (A1a), we have used the approximation

$$
\kappa \xi\left(\omega_{T} / 2+\alpha D-\alpha \xi / A\right) \approx \kappa\left(A \omega_{T} / 2+\alpha A D-\alpha \xi\right)
$$

in the forcing and damping terms since $\xi \approx A$.

In Bernsen et al. (2006), a finite element discretization is given and verified for the inviscid, unforced version of (A1) for complex shaped multiple connected domains. The generalized streamfunction and vorticity formulation (A1) is advantageous as it unifies several systems into one, such as the barotropic quasigeostrophic, and rigid-lid equations. A third order Runge-Kutta discretization in time and second, third or fourth order discretizations in 
space are implemented and available for use. Without forcing and dissipation, discrete energy conservation is guaranteed in space, while the discrete enstrophy decays for the upwind numerical flux and is conserved for the central flux, for infinitesimal time steps. The latter central flux is stable but yields small oscillations in combination with the third-order time integrator. When necessary, the circulation along the boundary can also be treated properly on the discrete level.

In extension to Bernsen et al. (2006), a discontinuous Galerkin method is used to find the weak formulation of (A1a). After we multiply (A1a) with an arbitrary test function $v_{h}$, integrate over the domain $\Omega$, and use numerical fluxes between interior elements and at the boundary elements, over each element $K$ the following weak formulation is obtained

$$
\begin{aligned}
\left(\partial_{t} \xi_{h} / A, v_{h}\right)_{K}= & \left(\xi_{h} \nabla^{\perp} \Psi_{h}, \nabla v_{h}\right)_{K}-\int_{\partial K} v_{h}^{-} \hat{F}\left(\xi_{h}^{+}, \xi_{h}^{-}, \nabla^{\perp} \Psi_{h} \cdot \hat{n}\right) \mathrm{d} \Gamma+ \\
& \left(v_{h}, \kappa\left(A \omega_{T} / 2+\alpha A D-\alpha \xi_{h}\right)\right)_{K},
\end{aligned}
$$

where the numerical flux $\hat{F}$ is replacing $\xi U_{n}$, in which $U_{n}$ is the component of the transport velocity $\mathbf{U}$ normal to an element face $\partial K ; \xi_{h}^{-}$and $\xi_{h}^{+}$are the limit values of the vorticity just in- and outside an element face; likewise $v_{h}^{-}$is the limit value of the test function just inside the element; and, $\partial_{t}=\partial / \partial_{t}$. Moreover, $(\cdot, \cdot)_{K}$ is the $L_{2}$-inner product over element domain $K$. In contrast, a continuous Galerkin finite element is used to find the weak formulation of (A1c); we multiply (A1c) with an arbitrary test function $w_{h}$, integrate over the domain $\Omega$, and use proper boundary conditions, to obtain

$$
\left(\sqrt{A} \nabla \Psi_{h}, \sqrt{A} \nabla w_{h}\right)_{\Omega}+\left(\sqrt{B} \Psi_{h}, \sqrt{B} w_{h}\right)_{\Omega}=-\left(\xi_{h} / A, w_{h}\right)_{\Omega}+\int_{\Omega} D w_{h} \mathrm{~d} \Omega+\left.w_{h}\right|_{\partial \Omega} \mathcal{C}
$$


with circulation

$$
\mathcal{C}=\int_{\partial \Omega} A \vec{U} \cdot \hat{\tau} \mathrm{d} \Gamma
$$

for the case $B>0$ with $\mathrm{d} \Gamma$ a line element and $\vec{\tau}$ the unit tangential vector along $\partial \Omega$. In case $B=0$, the streamfunction $\left.\Psi_{h}\right|_{\partial \Omega}=0$ and then the circulation is not required, in the singlyconnected domain used here. Subscripts $(\cdot)_{h}$ denote that test functions and variables are approximations in the appropriate function spaces, see Bernsen et al. (2006). These spaces are chosen specifically to guarantee energy conservation; that is, the space of functions for the discontinuous part of the discretization must cover the space of functions of the continuous part of the discretization. We have calculated several modal solutions as linear exact and asymptotic test solutions for the nonlinear numerical model.

b. Normal mode numerical tests

\section{1) Linear FREe AND FORCED-Dissipative Planetary Rossby MODES}

A free Rossby mode in a cylindrical domain of radius $R$ satisfies the linearized version of system (6) with constant $H=H_{0}=1, f=1+\beta y$, and $\kappa=0$, or (A1) with $A=1 / H_{0}$, $B=0, D=1+\beta y=D_{0}+\beta y$ and $\kappa=0$. A linear modal solution reads

$$
\Psi_{l i n r}(r, \theta, t)=b_{m} J_{m}\left(k_{l m} r\right) \cos \left(\beta r \cos \theta /\left(2 \omega_{l m}\right)+m \theta+\omega_{l m} t\right),
$$

where $b_{m}$ is the amplitude, $J_{m}$ the Bessel function of the first kind, $r$ the radius and $\theta$ the azimuthal angle, and frequency

$$
\omega_{l m}= \pm 0.5 \beta / k_{l m}
$$


the boundary condition is satisfied since $k_{l m}$ are the zeroes of $J_{m}\left(k_{l m} R\right)=0$ given the azimuthal mode number $m$ (with $l=1,2, \ldots, \infty)$. A comparison between the linear solution (A5) for $m=0$ is made with the nonlinear numerical solution initialized by the exact $\xi(x, y, 0)$, given the initial streamfunction $\Psi(x, y, 0)=\Psi_{l i n r}(r, \theta, 0)$. An approximate $\Psi_{h}(x, y, 0)$ is then calculated numerically. Good agreement between the linear exact and the nonlinear numerical solutions is found. We numerically also calculated the linear forceddissipative response of planetary Rossby modes with $\beta=0.3125$ as a representative laboratory value and $H_{1}=H_{2}=R$. Some of the first few frequencies of inviscid free modes are

$$
\omega_{1 m}=0.0650,0.0408,0.0304,0.0245,0.0206,
$$

and correspond to solutions (A5), The larger resonant frequencies match finely with the free-wave frequencies (A7).

\section{2) LiNEAR FREE SHELF MODE}

In the nonlinear numerical simulations, we wish to avoid a discontinuous profile of $A=$ $A(x, y)$ or the depth $A=1 / H$. Instead of a discontinuous step in the depth, we consider the continuous axisymmetric depth profile

$$
H(r, \theta)=\left\{\begin{array}{cc}
H_{1} & r \geq R_{s}+\epsilon \\
H_{2}+\frac{1}{2}\left(H_{1}-H_{2}\right)\left(r-R_{s}+\epsilon\right) / \epsilon & R_{s}-\epsilon<r<R_{s}+\epsilon \\
H_{2} & r \leq R_{s}-\epsilon
\end{array}\right.
$$

with $H_{1}<H_{2}$, the shelf break radius $R_{s}<R$ where the depth changes suddenly, and $\epsilon \ll 1$. A matched asymptotic solution (cf. Bokhove and Vanneste (2001)) to the linearized version 
of (6) is sought for $D=f_{0}=1, B=0$ and $A=1 / H$, i.e. a solution of:

$$
\partial_{t}\left(\frac{1}{r} \frac{\partial}{\partial r}\left(\frac{r}{H} \frac{\partial \Psi}{\partial r}\right)+\frac{1}{r^{2}} \frac{\partial^{2} \Psi}{\partial \theta^{2}}\right)+\frac{1}{r}\left(\frac{\partial \Psi}{\partial r} \frac{\partial H^{-1}}{\partial \theta}-\frac{\partial \Psi}{\partial \theta} \frac{\partial H^{-1}}{\partial r}\right)=0 .
$$

Outer solutions in the regions $r \leq R_{s}-\epsilon$ and $r \geq R_{s}+\epsilon$ will satisfy the linearized equation (A9) with depth (A8) in the step-shelf limit $\epsilon \rightarrow 0$. Inner solutions are valid in the transition region $R_{s}-\epsilon<r<R_{s}+\epsilon$, and a suitable sum of inner and outer solutions will provide the entire asymptotic solution.

Outer solutions satisfy, to all orders, $\nabla^{2} \Psi=0$ with $\Psi(R, \theta, t)=0$; hence, it concerns the real part of

$$
\left\{\begin{array}{cc}
\Psi_{1}(r, \theta, t)=\sum_{m \neq 0} a_{m}\left((r / R)^{m}-(R / r)^{m}\right) e^{i(m \theta+\omega t)} & r>R_{s} \\
\Psi_{2}(r, \theta, t)=\sum_{m \neq 0} c_{m} r^{|m|} e^{i(m \theta+\omega t)} & r \leq R_{s}
\end{array}\right.
$$

with coefficients $a_{m}, c_{m}$, frequency $\omega$, and azimuthal wave number $m$.

For the inner solution, a stretched coordinate $\zeta=\left(r-R_{s}\right) / \epsilon$ is introduced. Subsequently, the inner expansion

$$
\Psi=\Psi\left(R_{s}, \theta\right)+\epsilon \phi^{(1)}(\zeta, \theta)+\ldots
$$

is substituted in (A9) and evaluated at leading order in $\epsilon$; giving the leading-order equation

$$
i \omega \frac{\partial}{\partial \zeta}\left(\frac{1}{H} \frac{\partial \phi^{(1)}}{\partial \zeta}\right)-\frac{1}{R_{s}} \frac{\partial \Psi}{\partial \theta}\left(R_{s}, \theta\right) \frac{\partial H^{-1}}{\partial \zeta}=0
$$

Since the outer expansion in the inner region satisfies

$$
\Psi(r, \theta)=\Psi\left(R_{s}, \theta\right)+\epsilon \zeta \frac{\partial \Psi}{\partial r}\left(R_{s}, \theta\right)+\ldots
$$

and the inner one (A11), we obtain together with the continuity requirement the following inner boundary conditions at $\zeta= \pm 1$ :

$$
\Psi_{1}\left(R_{s}, \theta\right)=\Psi_{2}\left(R_{s}, \theta\right) \quad \text { and } \quad \frac{\partial \Psi}{\partial r}\left(R_{s}^{ \pm}, \theta\right)=\left.\frac{\partial \phi^{(1)}}{\partial \zeta}\right|_{\zeta= \pm 1} .
$$


Integration of (A12) from $\zeta=-1$ to $\zeta=1$ using (A13) yields the dispersion relation for the outer expansion

$$
\omega\left(\frac{1}{H_{1}} \frac{\partial \Psi_{1}}{\partial r}\left(R_{s}, \theta\right)-\frac{1}{H_{2}} \frac{\partial \Psi_{2}}{\partial r}\left(R_{s}, \theta\right)\right)-\frac{m}{R_{s}} \Psi\left(R_{s}, \theta\right)\left(\frac{1}{H_{1}}-\frac{1}{H_{2}}\right)=0 .
$$

In rewritten form and by using $\Psi_{1}\left(R_{s}, \theta\right)=\Psi_{2}\left(R_{s}, \theta\right)$ and (A10), the outer solutions therefore become

$$
\begin{aligned}
& \Psi(r, \theta, t)=\left\{\begin{array}{cc}
\Psi_{1}(r, \theta, t)=\sum_{m \neq 0} a_{m}\left(\frac{(r / R)^{m}-(R / r)^{m}}{\left(R_{s} / R\right)^{m}-\left(R / R_{s}\right)^{m}}\right) e^{i(m \theta+\omega t)} & r>R_{s} \\
\Psi_{2}(r, \theta, t)=\sum_{m \neq 0} a_{m} \frac{r}{R_{s}}|m| e^{i(m \theta+\omega t)} & r>R_{s}
\end{array}\right. \\
& \omega=\frac{\left(H_{2}-H_{1}\right)\left(\left(\frac{R_{s}}{R}\right)^{m}-\left(\frac{R}{R_{s}}\right)^{m}\right)}{H_{2}\left(\left(\frac{R_{s}}{R}\right)^{m}+\left(\frac{R}{R_{s}}\right)^{m}\right)-H_{1} \frac{|m|}{m}\left(\left(\frac{R_{s}}{R}\right)^{m}-\left(\frac{R}{R_{s}}\right)^{m}\right)} .
\end{aligned}
$$

A first integration of (A12) from $\zeta=-1$ to $\zeta<1$ or $\zeta>-1$ to $\zeta=1$, using (A13), followed by a second integration, gives

$$
\phi^{(1)}=\frac{\partial \Psi_{2}}{\partial r}\left(R_{s}, \theta\right) \zeta+\left(\frac{1}{H_{2}} \frac{\partial \Psi_{2}}{\partial r}\left(R_{s}, \theta\right)-\frac{m}{R_{s} \omega H_{2}} \Psi\left(R_{s}, \theta\right)\right) \frac{\left(H_{1}-H_{2}\right)}{4}(\zeta+1)^{2}+c_{1} .
$$

At $\zeta=-1$, using (A16), we find therefore

$$
\phi^{(1)}(-1, \theta)=-\frac{\partial \Psi_{2}}{\partial r}\left(R_{s}, \theta\right)+c_{1}
$$

and, likewise, at $\zeta=1$,

$$
\phi^{(1)}(1, \theta)=\frac{\partial \Psi_{1}}{\partial r}\left(R_{s}, \theta\right)+c_{1}
$$

where we used the dispersion relation (A14) to find the relation at $\zeta=1$. Considering the inner and outer expansion in the inner region, we choose $c_{1}=0$; it also explains why the outer solution is chosen to hold at all orders. 
The uniformly valid matched asymptotic solution consists of the mean of inner and outer solutions (A16) with $c_{1}=0$ and (A15)

$$
\Psi_{\text {Uniform }}=\left\{\begin{array}{cc}
\Psi_{1} & r \geq R_{s}+\epsilon \\
\Psi\left(R_{s}, \theta\right)+\frac{1}{2}\left(r-R_{s}\right) \frac{\partial \Psi}{\partial r}\left(R_{s}, \theta\right)+\frac{1}{2}\left(\frac{\partial \Psi_{2}}{\partial r}\left(R_{s}, \theta\right)\left(r-R_{s}\right)+\right. & R_{s}-\epsilon< \\
\left.\left(\frac{1}{H_{2}} \frac{\partial \Psi_{2}}{\partial r}\left(R_{s}, \theta\right)-\frac{m}{R_{s} \omega H_{2}} \Psi\left(R_{s}, \theta\right)\right) \frac{H_{1}-H_{2}}{4 \epsilon}\left(r-R_{s}+\epsilon\right)^{2}\right) & r<R_{s}+\epsilon \\
\Psi_{2} & r \leq R_{s}-\epsilon
\end{array} .\right.
$$

We initialized the nonlinear numerical simulation with the potential vorticity based on the asymptotic solution (A19) of the streamfunction $\Psi=\Psi_{\text {Uniform }}$ at $t=0$. The agreement between the asymptotic linear solution and the nonlinear numerical solution of the streamfunction was quite reasonable.

\section{3) ForCed-Dissipative HYBRID-ShELF MODES}

In the nonlinear numerical simulations, we use $A=A(x, y)=1 / H(x, y), D(x, y)=f_{0}=$ 1. Instead of a discontinuous step in the depth, we consider a depth profile

$$
H(r, \theta)=\left\{\begin{array}{cc}
H_{1 s}=H_{1}-s_{1} y & r>R_{s}+\epsilon \\
H_{2 s}+\frac{1}{2}\left(H_{1 s}-H_{2 s}\right)\left(r-R_{s}+\epsilon\right) / \epsilon & R_{s}-\epsilon<r<R_{s}+\epsilon \\
H_{2 s}=H_{2}-s_{2} y & r<R_{s}-\epsilon
\end{array}\right.
$$

with $H_{1}<H_{2}, s_{i}=\beta H_{i}$ and $\epsilon \ll 1$, for $i=1,2$. The simulated streamfunction fields displayed in Fig. 18 over forcing period 19 are seen to equal the ones in forcing period 20 in Fig. 16. Parameter values are $\sigma=0.0613, \kappa=0.0042, \epsilon=0.0314$, and $\Delta \theta=2 \pi$. 


\section{REFERENCES}

Bernsen, E., O. Bokhove, and J. van der Vegt, 2006: A (dis)continuous finite element model for generalized 2d vorticity dynamics. J. Comp. Phys., 212, 719-747.

Bokhove, O., 1999: Forced-dissipative response for coupled planetary rossby and topographic shelf modes in homogeneous, cylindrical oceans. In: Twelfth A.M.S. Conference Proceedings on Atmospheric and Oceanic Fluid Dynamics, 104-107.

Bokhove, O. and E.R. Johnson, 1999: Hybrid coastal and interior modes for two-dimensional flow in a cylindrical ocean. J. Phys. Ocean., 29, 93-118.

Bokhove, O. and J. Vanneste, 2001: Homogenizing coastal canyons. Notes and personal communication.

Greenspan, H., 1968: The theory of rotating fluids. Cambridge University Press, 354 pp.

Kelly, K., R. Beardsley, R. Limeburner, K. Brink, J. Paduan, and T. Chereskin, 1998: Variability of the near-surface eddy kinetic energy in the california current based on altimetric, drifter, and moored current data. J. Geophys. Res., 103, 13 067-13 083.

LaCasce, J., O. Nost, and P. Isachsen, 2008: Asymmetry of free circulations in closed ocean gyres. J. Phys. Ocean., 38, 517-526.

Pedlosky, J., 1987: Geophysical Fluid Dynamics. Springer, 710 pp.

Pedlosky, J., 1996: Ocean Circulation Theory. Springer, 453 pp. 
Pedlosky, J. et al., 2007: Boundary layers, GFD Volume 2007, Lecture 1. Woods Hole Oceanographic Institution, 1-11 pp.

Weijer, W., 2008: Normal modes of the Mascarene Basin. Deep Sea Research Part I: Oceanographic Research Papers, 55, 128-136.

Wunsch, C., 2004: Vertical mixing, energy, and the general circulation. Ann. Rev. Fluid. Mech., 36, 281-314. 


\section{APPENDIX B}

\section{Vorticity equation with Ekman forcing and dissipation}

Consider the vertical component of the vorticity vector for the incompressible NavierStokes equations in three dimensions ${ }^{1}$

$$
\partial_{t} \omega+\left(\mathbf{v} \cdot \nabla_{H}\right) \omega+w \partial_{z} \omega+2 v \partial_{y} \Omega_{r}+2 u \partial_{x} \Omega_{r}=\left(2 \Omega_{r}+\omega\right) \partial_{z} w+\nu \nabla_{3}^{2} \omega
$$

with the vertical vorticity component $\omega=\partial_{x} v-\partial_{y} u, \nabla_{H}=\left(\partial_{x}, \partial_{y}\right)^{T}, \nabla_{3}=\left(\partial_{x}, \partial_{y}, \partial_{z}\right)^{T}$, in a cylindrical domain with bottom topography, and with rotation $\Omega_{r}$ aligned along the $z$-axis of the cylinder. This equation for the vertical vorticity is simplified as follows:

(a) internal and side-wall friction is ignored relative to the Ekman damping and driving because focus is put on large-scale driven modes;

(b) outside top and bottom Ekman boundary layers the dynamics are assumed inviscid and uniform in $z$ with depth $H=H(x, y)$, such that $\omega \approx \omega(x, y, t)$ and horizontal transport velocity $H \mathbf{v}=\nabla^{\perp} \Psi$ is rotational at leading order; and, finally,

(c) the absolute vorticity $2 \Omega_{r}+\omega$ in the vertical direction is approximated as $2 \Omega_{r}$ for the Ekman forcing and viscous terms.

Under these assumptions (a) and (b), integration of (B1) from just above the bottom boundary layer to just below the top boundary layer yields as an intermediate step

$$
H \partial_{t} \omega+J\left(\Psi, 2 \Omega_{r}+\omega\right)=\left(2 \Omega_{r}+\omega\right)\left(w_{T}-w_{B}\right)
$$

\footnotetext{
${ }^{1}$ This Appendix is not part of a journal publication, but is solely supplied as clarification, for the authors.
} 
with top and bottom values $w_{T}$ and $w_{B}$ of the vertical velocity just below and above the respective Ekman boundary layers, and Jacobian $J\left(F_{1}, F_{2}\right) \equiv \partial_{x}\left(F_{1}\right) \partial_{y}\left(F_{2}\right)-\partial_{x}\left(F_{2}\right) \partial_{y}\left(F_{1}\right)$ for two arbitrary functions $F_{1}=F_{1}(x, y, t)$ and $F_{2}=F_{2}(x, y, t)$.

The next step is to establish approximate relationships for these vertical Ekman velocities using asymptotic analysis of the flow in the boundary layer (Pedlosky 1987; Pedlosky et al. 2007). Consider the scaled three-dimensional incompressible Navier-Stokes equations

$$
\begin{aligned}
\epsilon \frac{D \mathbf{v}}{D t}+\mathbf{v}^{\perp} & =-\nabla_{H} P+\frac{E}{2}\left(\nabla_{H}^{2} \mathbf{v}+\frac{1}{\delta^{2}} \frac{\partial^{2} \mathbf{v}}{\partial z^{2}}\right) \\
\delta^{2} \epsilon \frac{D w}{D t} & =-\partial_{z} P+\delta^{2} \frac{E}{2}\left(\nabla_{H}^{2} w+\frac{1}{\delta^{2}} \frac{\partial^{2} w}{\partial z^{2}}\right) \\
\boldsymbol{\nabla}_{H} \mathbf{v}+\partial_{z} w & =0
\end{aligned}
$$

with material derivative $D / D t$, modified pressure $P=p+\epsilon z / F^{2}$, constant density $\rho$, and Froude number $F$. The non-dimensional numbers involved are the Rossby number $\epsilon=$ $U /\left(2 \Omega_{r} L\right)$, Ekman number $E=\nu /\left(\Omega_{r} L^{2}\right)$, and the aspect ratio $\delta=D / L=W / U$ between horizontal $L, U$ and vertical $D, W$ length and velocity scales. Pressure was scaled as $p^{\star}=P_{0} p$ with $P_{0}=2 \rho \Omega_{r} L U$.

In the top and bottom boundary layers, we take $\delta=\sqrt{E}$. In the outer region $\delta=O(1)$ and we find geostrophic balance at leading order in $\epsilon$ and $E$; for simplicity the uni-directional case is considered with $u=U_{I}(y), v=0$ and $P=-\int^{y} U_{I}(\tilde{y}) \mathrm{d} \tilde{y}$. The boundary layer equations arising at leading order can be summarized to govern

$$
\frac{\partial^{2} \Lambda}{\partial z^{2}}=2 i \Lambda
$$

with $\Lambda=\left(u-U_{I}\right)+i v$. At a flat bottom $z=0$, one finds

$$
\Lambda=-U_{I} \cos z e^{-z}+i U_{I} \sin z e^{-z}
$$


such that $u=v=0$ at $z=0$ and $u \rightarrow U_{I}$ for the outer region $z \gg 1$. The associated vertical velocity follows from integration $\partial_{z} w=-\partial_{y} v$, and yields

$$
w=-\frac{\mathrm{d} U_{I}}{\mathrm{~d} y} \frac{1}{2}\left(1-(\sin z+\cos z) e^{-z}\right)
$$

such that for $z \gg 1$ the outer value becomes $w_{B}=-(1 / 2) \mathrm{d} U_{I} / \mathrm{d} y \approx \omega / 2$. Hence, we have extrapolated $-\mathrm{d} U_{I} / \mathrm{d} y$ to generalize to the interior value $\omega=\omega(x, y, t)$ for the general timedependent case with $U_{I}=U_{I}(x, y, t)$. For a sloping bottom $z=b(x, y)=H_{0}-H(x, y)$, these results extend further to

$$
w_{B}=\omega / 2-\mathbf{v} \cdot \nabla H
$$

In a similar way, by using slightly different boundary conditions, we find in the top boundary layer at $z=H_{0}$ that, for rigid lid driven with velocity $u=U_{T}$,

$$
\Lambda=-U_{I} \cos \left(z-H_{0}\right) e^{z-H_{0}}+U_{T} \cos \left(z-H_{0}\right) e^{z-H_{0}}+i\left(U_{I}-U_{T}\right) \sin \left(z-H_{0}\right) e^{z-H_{0}}
$$

such that $u=U_{T}$ and $v=0$ at $z=H_{0}$ and $u \rightarrow U_{I}$ for $0 \ll z \ll H_{0}$. The vertical velocity below this boundary layer then satisfies

$$
w_{T}=\omega_{T} / 2-\omega / 2
$$

in extension to using $-\mathrm{d} U_{T}(x, y, t) / \mathrm{d} y$ and $\mathrm{d} U_{I}(x, y, t) / \mathrm{d} y$. In dimensional form, the expressions

$$
w_{B}=\left(D_{E} / 2\right) \omega \quad \text { and } \quad w_{T}=\left(D_{E} / 2\right)\left(\omega_{T}-\omega\right)
$$

are substituted into (B2), with Ekman depth $D_{E}=\sqrt{\nu / \Omega_{r}}$. We obtain therefore

$$
H \partial_{t} \omega+H J\left(\Psi,\left(2 \Omega_{r}+\omega\right) / H\right)=\left(2 \Omega_{r}+\omega\right) D_{E}\left(\omega_{T} / 2-\omega\right)
$$

with assumptions (b) and (c) this simplifies to the starting equation (1) in the main text. 


\section{List of Figures}

1 Sketch of laboratory domain with abrupt shelf topography, and deep interiorocean and shallow-shelf slopes mimicking $\beta$ (Bokhove and Johnson 1999). . . 31

2 Linear forced-dissipative response for topographic Rossby-shelf modes displayed as the $L_{\infty}$-norm of $|\Psi|$ against 500 forcing frequencies $\sigma$. Parameter values $\omega_{T}^{\max }=2 \sigma \Delta \theta=2 \sigma 2 \pi$, and $\kappa=\sqrt{\nu / \Omega_{r}} / H_{0}=0.0042 . \ldots$.

$3 \quad$ Streamfunction field for Rossby-shelf modes over one forcing period $T$ nearby the maximum response $\sigma=0.0613$. Parameter values $\omega_{T}^{\max }=2 \sigma \Delta \theta=$

$2 \sigma 2 \pi$, and $\kappa=\sqrt{\nu / \Omega_{r}} / H_{0}=0.0042 \ldots \ldots \ldots \ldots$

4 Streamfunction field for Rossby-shelf modes over one forcing period $T$ at the maximum response $\sigma=0.0878 \ldots \ldots \ldots$. . . . . . . . . . . . 34

5 Dispersion relation of the free planetary Rossby mode of zeroth order in the radial direction, and the coastal shelf mode for $\beta=0.3125$; and $H_{1}=0.6 R$, $H_{2}=0.8 R$, and $R_{s}=0.8 R . \ldots \ldots \ldots \ldots \ldots$

6 Linear forced-dissipative response for topographic Rossby-shelf modes displayed as the $L_{\infty}$-norm of $|\Psi|$ against 500 forcing frequencies $\sigma$ for a smoothed shelf break of width $2 \epsilon=0.0628$ around $r=R_{s}$. Parameter values $\omega_{T}^{\max }=$ $2 \sigma \Delta \theta=2 \sigma 2 \pi$, and $\kappa=\sqrt{\nu / \Omega_{r}} / H_{0}=0.0042 \ldots \ldots \ldots$

$7 \quad$ Streamfunction field for Rossby-shelf modes over one forcing period $T$ at the maximum response $\sigma=0.0577$ for a smoothed shelf break. Parameter values $\omega_{T}^{\max }=2 \sigma \Delta \theta=2 \sigma 2 \pi$, and $\kappa=\sqrt{\nu / \Omega_{r}} / H_{0}=0.0042 \ldots \ldots . . .$.

8 Sketch of the laboratory set-up. . . . . . . . . . . . . . . 38 
9 Streak photography of hybrid-Rossby shelf modes at $t=0,7,14,28,35,42,49$ s for a forcing period of 51.3s (nondimensional $\sigma=0.0612$ ), and maximum rigid-lid excursion $\Delta \theta=\pi$. Exposure time was 4 s. . . . . . . . . . . . . . . .

10 Streak photography of hybrid-Rossby shelf modes at $t=0,7,14,28,35,42,49$ s for a forcing period of 51.3s (nondimensional $\sigma=0.0612$ ), and maximum rigid-lid excursion $\Delta \theta=2 \pi$. Exposure time was 2 s. . . . . . . . . . . . . . . . . . . . . 40

11 a) Streak photography of hybrid-Rossby shelf mode observed at $t=0$ s for a forcing period of 51.3s (nondimensional $\sigma=0.0612$ ), and maximum rigid-lid excursion $\Delta \theta=\pi$. Exposure time was $4 \mathrm{~s}$. b) Similarly, for the calculated linear solution; phase shift adjusted semi-optimally by eye. . . . . . . . . . . . . . . . .

12 a) Streak photography of hybrid-Rossby shelf mode observed at $t=0$ s for a forcing period of 51.3s (nondimensional $\sigma=0.0612$ ), and maximum rigid-lid excursion $\Delta \theta=2 \pi$. Exposure time was $2 \mathrm{~s}$. b) Similarly, for the calculated linear solution; phase shift adjusted semi-optimally by eye. . . . . . . . . . . . .

13 Streak photography of hybrid-Rossby shelf modes at $t=0,5,10,15,20,25,30,35 \mathrm{~s}$ for a forcing period of 36.1s (nondimensional $\sigma=0.0871$ ), and maximum rigid-lid excursion $\Delta \theta=2 \pi \ldots \ldots \ldots \ldots \ldots$

14 Streak photography of hybrid-Rossby shelf modes at $t=0,5,10,15,20,25,30,35 \mathrm{~s}$ for a forcing period of 36.1s (nondimensional $\sigma=0.0871$ ), and maximum rigid-lid excursion $\Delta \theta=\pi / 2 \ldots \ldots \ldots \ldots \ldots$

15 Energy and enstrophy versus time. . . . . . . . . . . . . . . . . . 45

16 Streamfunction over forcing period 20; $\sigma=0.0613, \kappa=0.0042, \epsilon=0.0314$, and $\Delta \theta=2 \pi$ 
17 Potential vorticity over forcing period 20; $\sigma=0.0613, \kappa=0.0042, \epsilon=0.0314$, and $\Delta \theta=2 \pi \ldots \ldots \ldots \ldots \ldots$

18 Streamfunction fields over forcing period $19 . \quad \ldots \ldots$. . . . . . . . . . . 48 

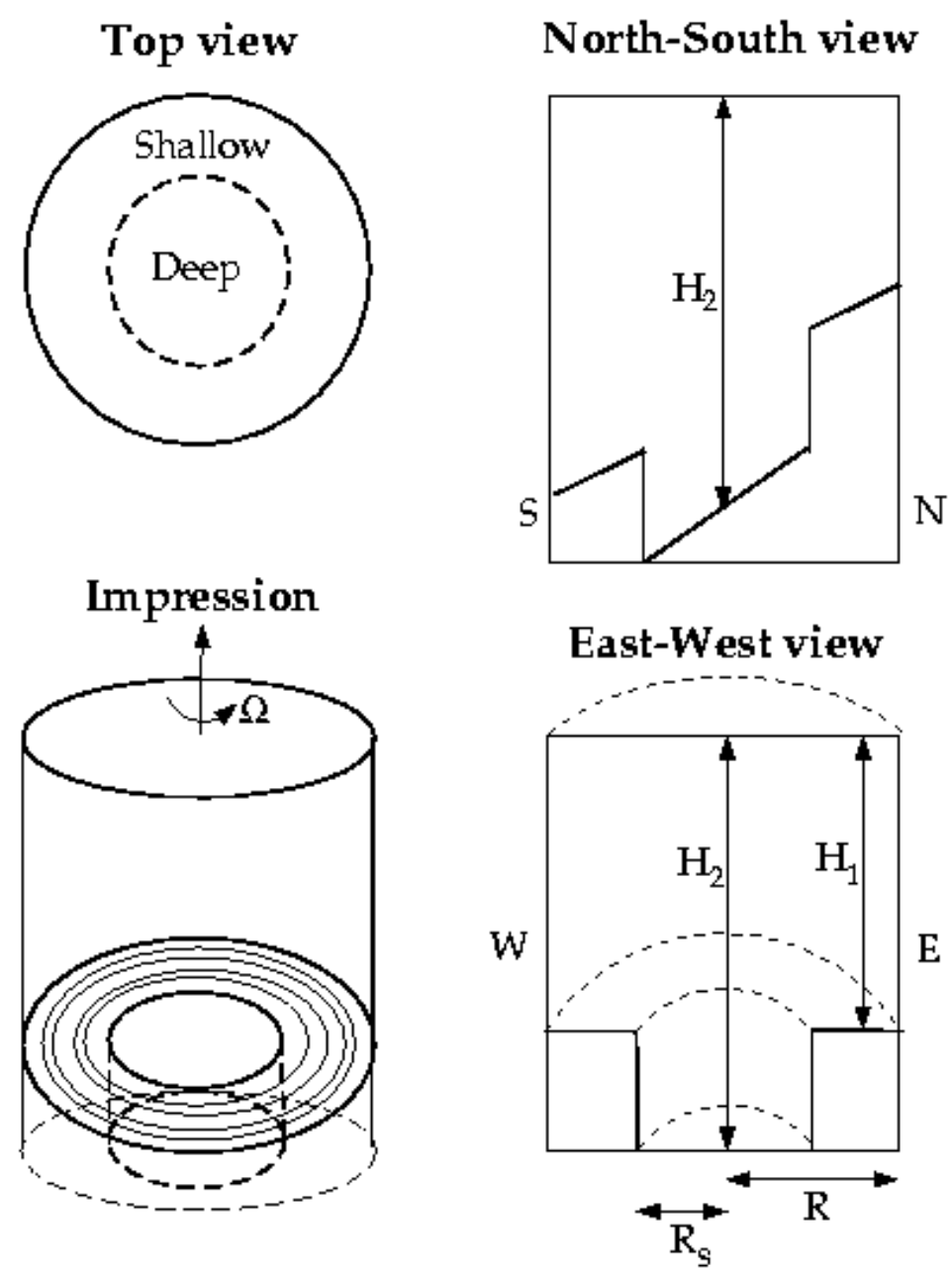

FIG. 1. Sketch of laboratory domain with abrupt shelf topography, and deep interior-ocean and shallow-shelf slopes mimicking $\beta$ (Bokhove and Johnson 1999). 


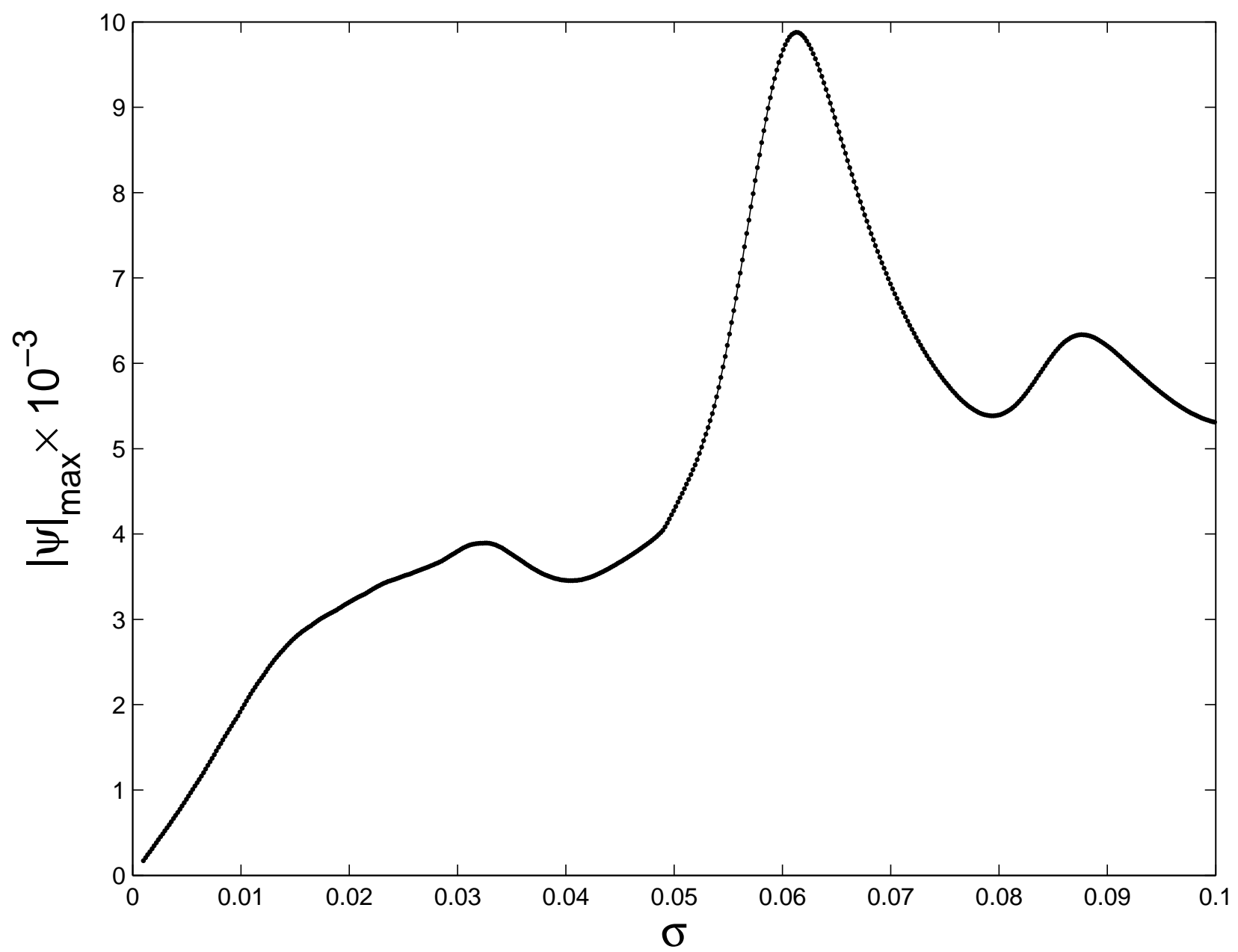

FIG. 2. Linear forced-dissipative response for topographic Rossby-shelf modes displayed as the $L_{\infty}-$ norm of $|\Psi|$ against 500 forcing frequencies $\sigma$. Parameter values $\omega_{T}^{\max }=2 \sigma \Delta \theta=$ $2 \sigma 2 \pi$, and $\kappa=\sqrt{\nu / \Omega_{r}} / H_{0}=0.0042$. 

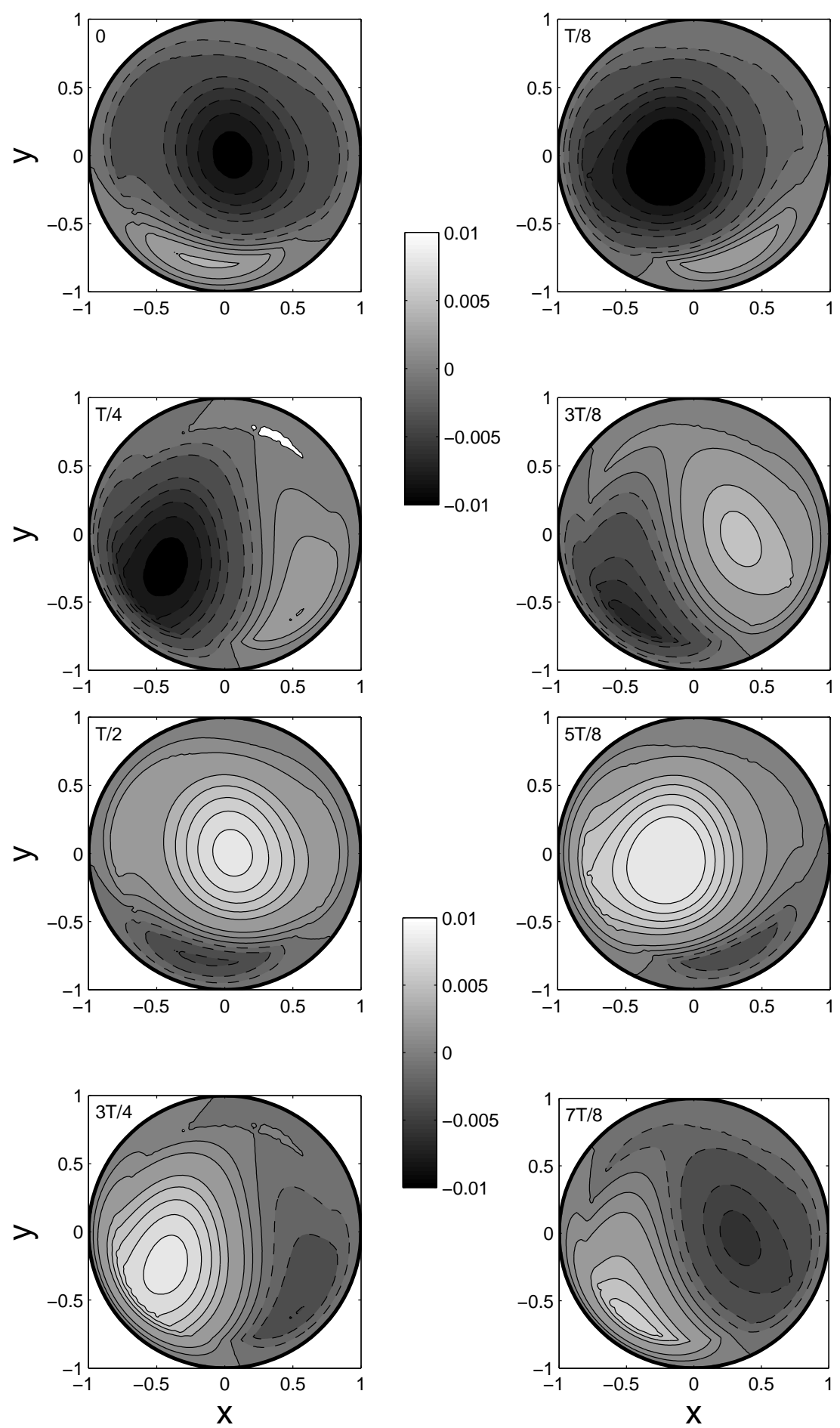

FIG. 3. Streamfunction field for Rossby-shelf modes over one forcing period $T$ nearby the maximum response $\sigma=0.0613$. Parameter values $\omega_{T}^{\max }=2 \sigma \Delta \theta=2 \sigma 2 \pi$, and $\kappa=$ $\sqrt{\nu / \Omega_{r}} / H_{0}=0.0042$. 

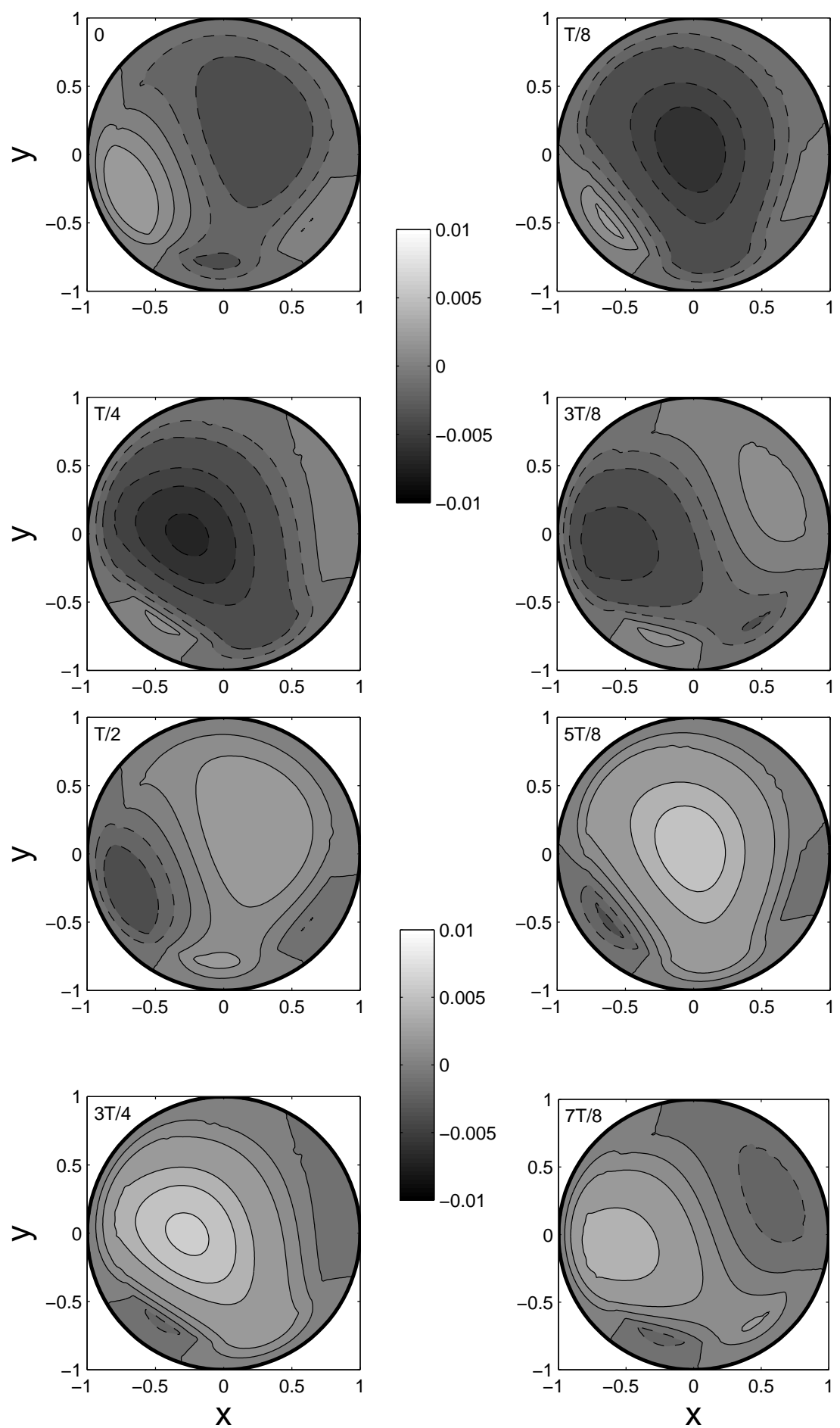

FIG. 4. Streamfunction field for Rossby-shelf modes over one forcing period $T$ at the maximum response $\sigma=0.0878$. 


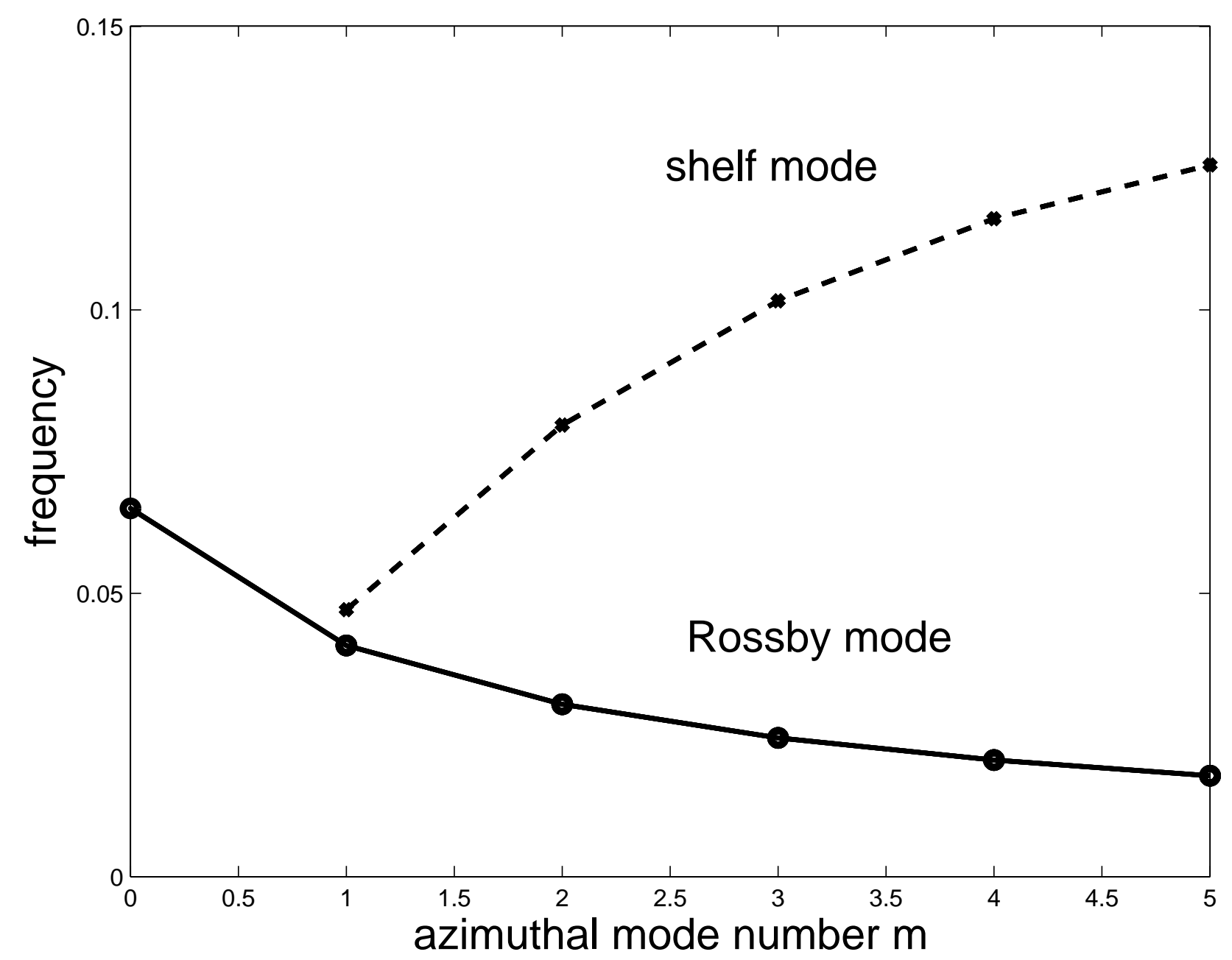

FIG. 5. Dispersion relation of the free planetary Rossby mode of zeroth order in the radial direction, and the coastal shelf mode for $\beta=0.3125$; and $H_{1}=0.6 R, H_{2}=0.8 R$, and $R_{s}=0.8 R$ 


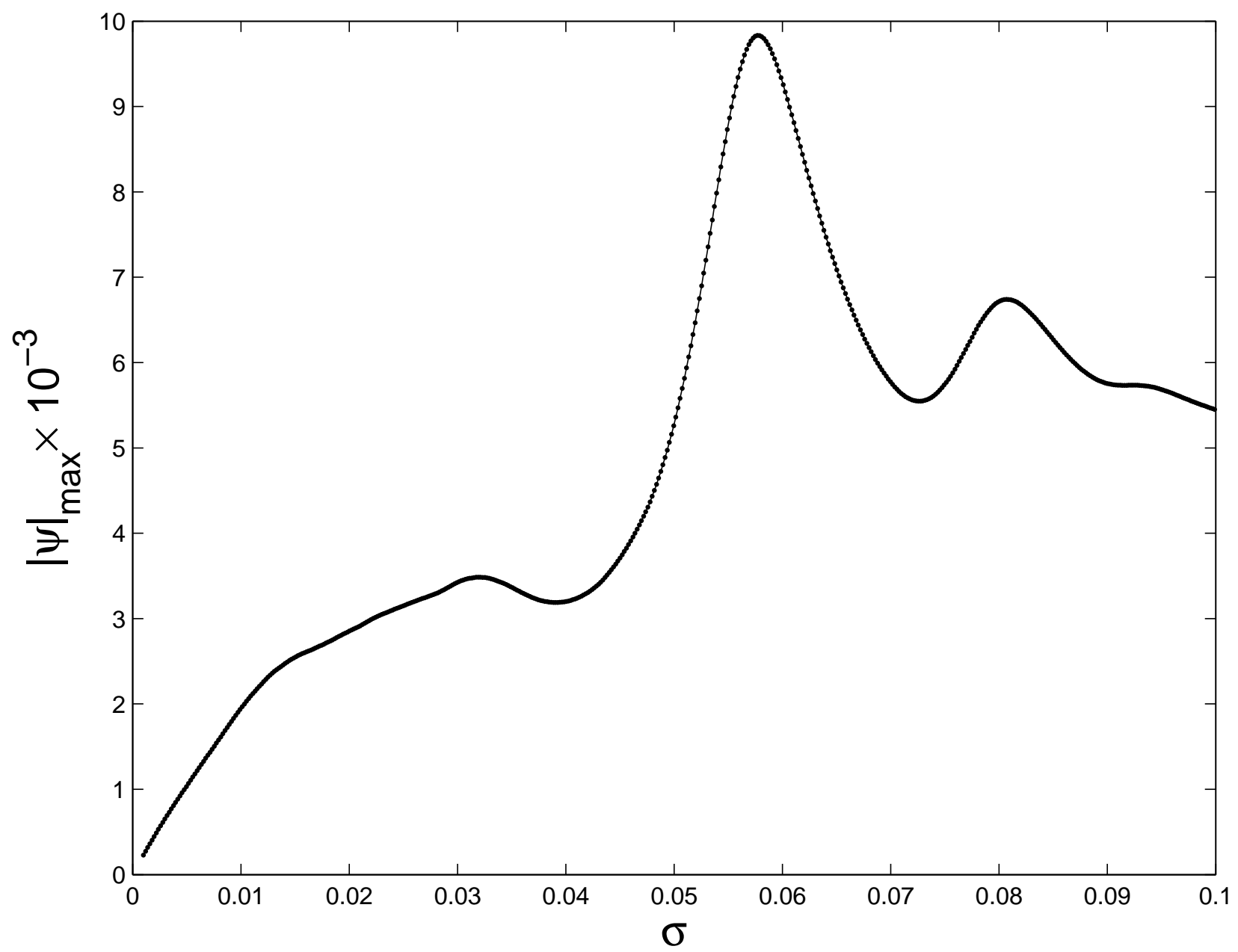

FIG. 6. Linear forced-dissipative response for topographic Rossby-shelf modes displayed as the $L_{\infty}$-norm of $|\Psi|$ against 500 forcing frequencies $\sigma$ for a smoothed shelf break of width $2 \epsilon=0.0628$ around $r=R_{s}$. Parameter values $\omega_{T}^{\max }=2 \sigma \Delta \theta=2 \sigma 2 \pi$, and $\kappa=$ $\sqrt{\nu / \Omega_{r}} / H_{0}=0.0042$. 

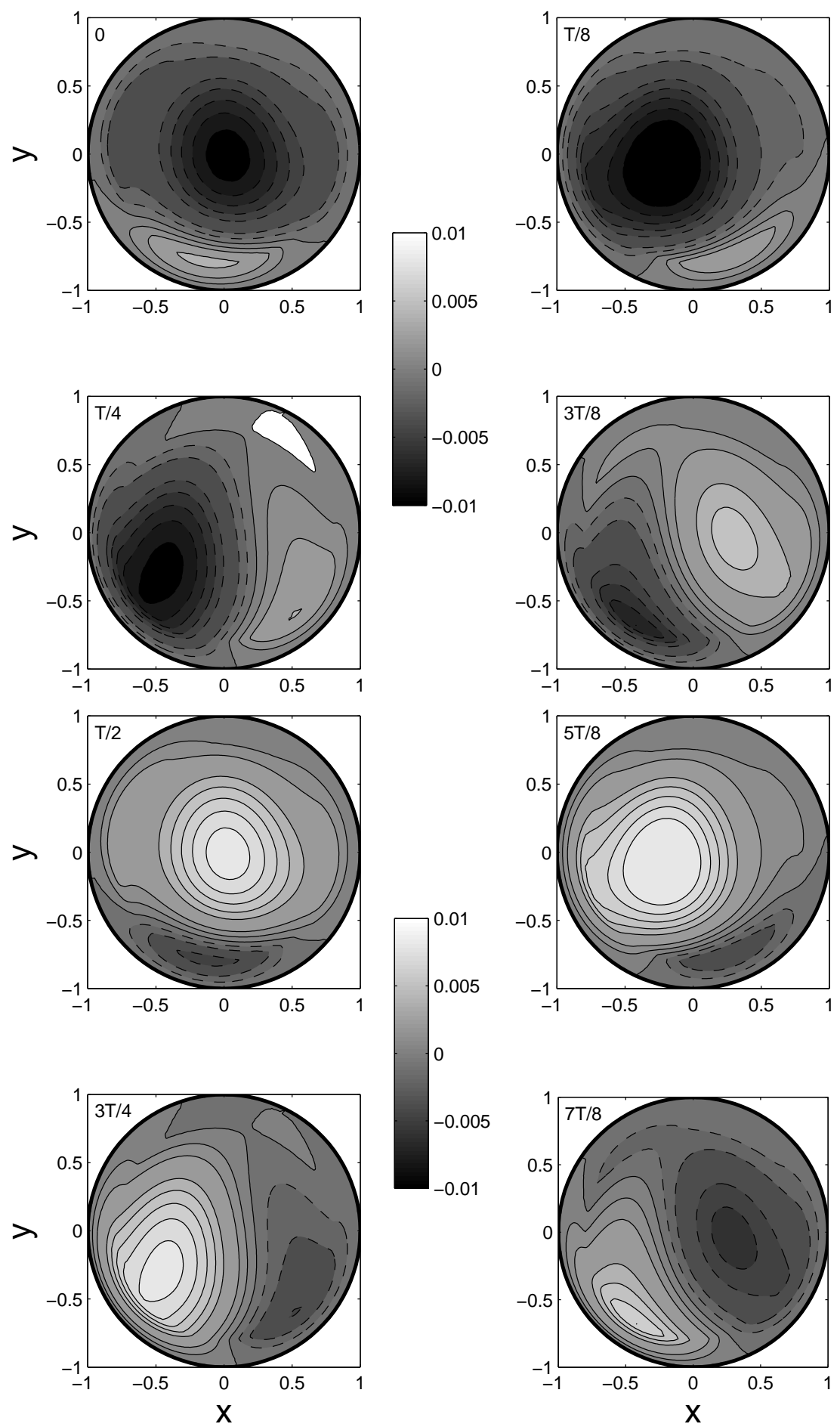

FIG. 7. Streamfunction field for Rossby-shelf modes over one forcing period $T$ at the maximum response $\sigma=0.0577$ for a smoothed shelf break. Parameter values $\omega_{T}^{\max }=2 \sigma \Delta \theta=$ $2 \sigma 2 \pi$, and $\kappa=\sqrt{\nu / \Omega_{r}} / H_{0}=0.0042$. 


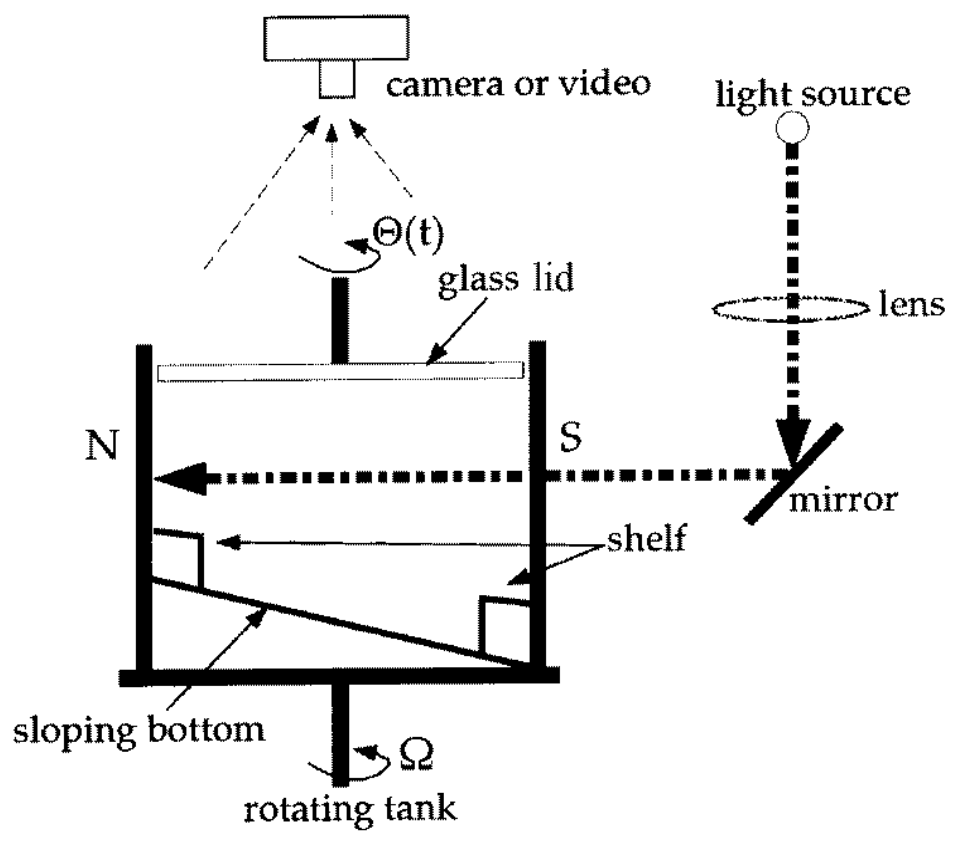

FIG. 8. Sketch of the laboratory set-up. 

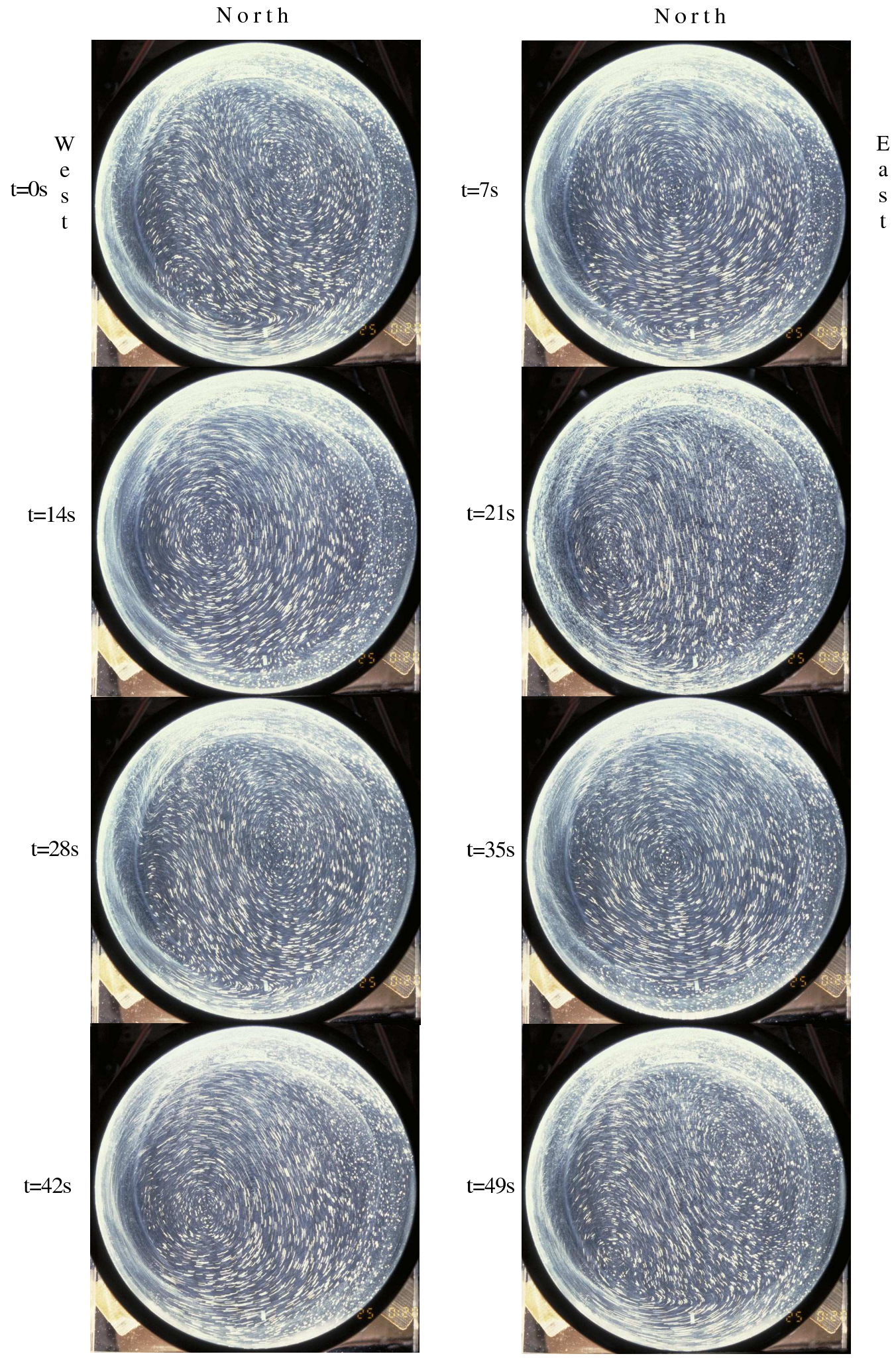

FIG. 9. Streak photography of hybrid-Rossby shelf modes at $t=0,7,14,28,35,42,49$ s for a forcing period of 51.3s (nondimensional $\sigma=0.0612$ ), and maximum rigid-lid excursion $\Delta \theta=\pi$. Exposure time was $4 \mathrm{~s}$. 

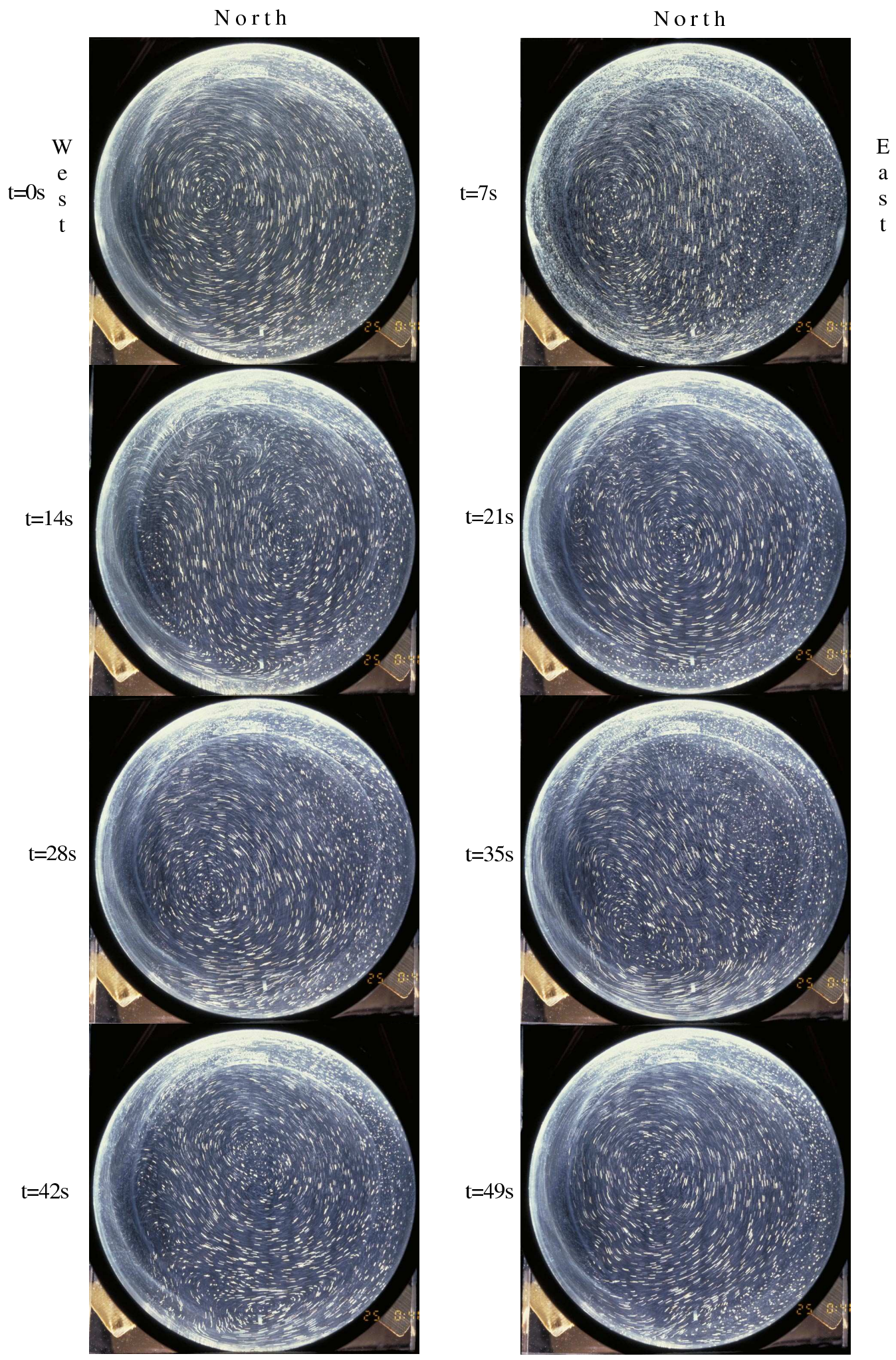

FIG. 10. Streak photography of hybrid-Rossby shelf modes at $t=0,7,14,28,35,42,49$ s for a forcing period of 51.3s (nondimensional $\sigma=0.0612$ ), and maximum rigid-lid excursion $\Delta \theta=2 \pi$.

Exposure time was $2 \mathrm{~s}$. 

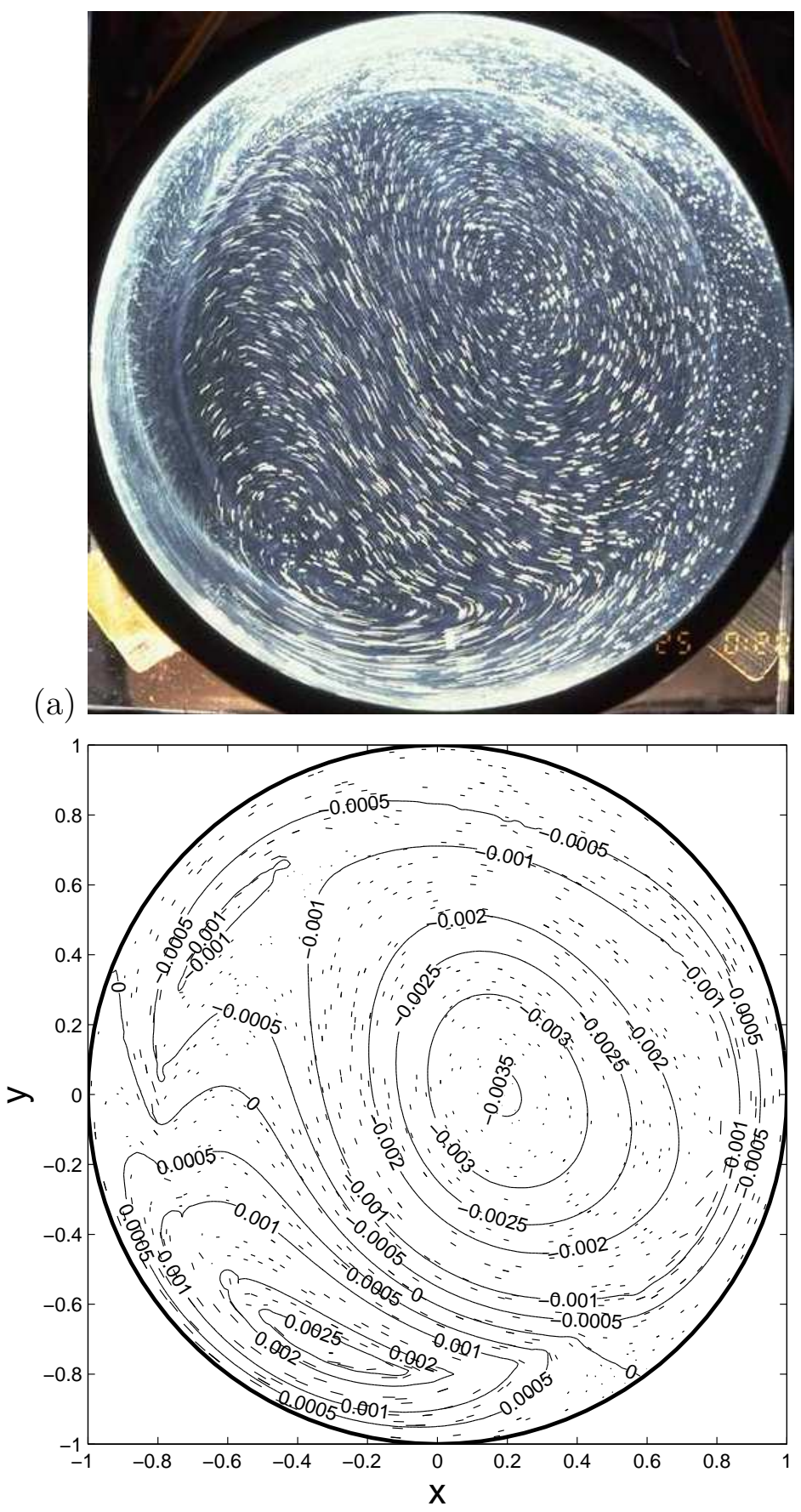

(b)

FIG. 11. a) Streak photography of hybrid-Rossby shelf mode observed at $t=0$ s for a forcing period of 51.3s (nondimensional $\sigma=0.0612$ ), and maximum rigid-lid excursion $\Delta \theta=\pi$. Exposure time was 4s. b) Similarly, for the calculated linear solution; phase shift adjusted semi-optimally by eye. 

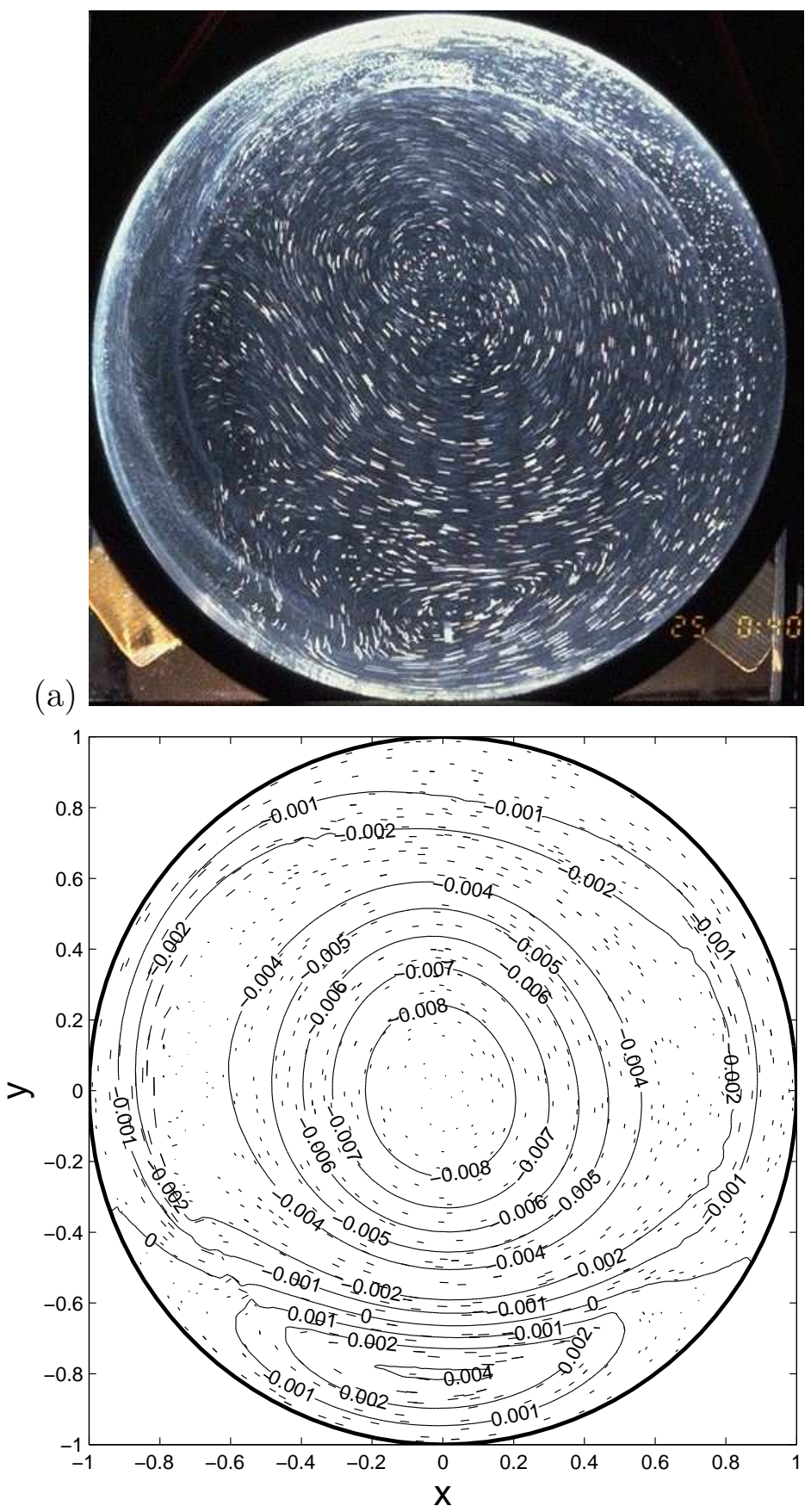

(b)

FIG. 12. a) Streak photography of hybrid-Rossby shelf mode observed at $t=0$ s for a forcing period of 51.3s (nondimensional $\sigma=0.0612$ ), and maximum rigid-lid excursion $\Delta \theta=2 \pi$. Exposure time was 2 s. b) Similarly, for the calculated linear solution; phase shift adjusted semi-optimally by eye. 

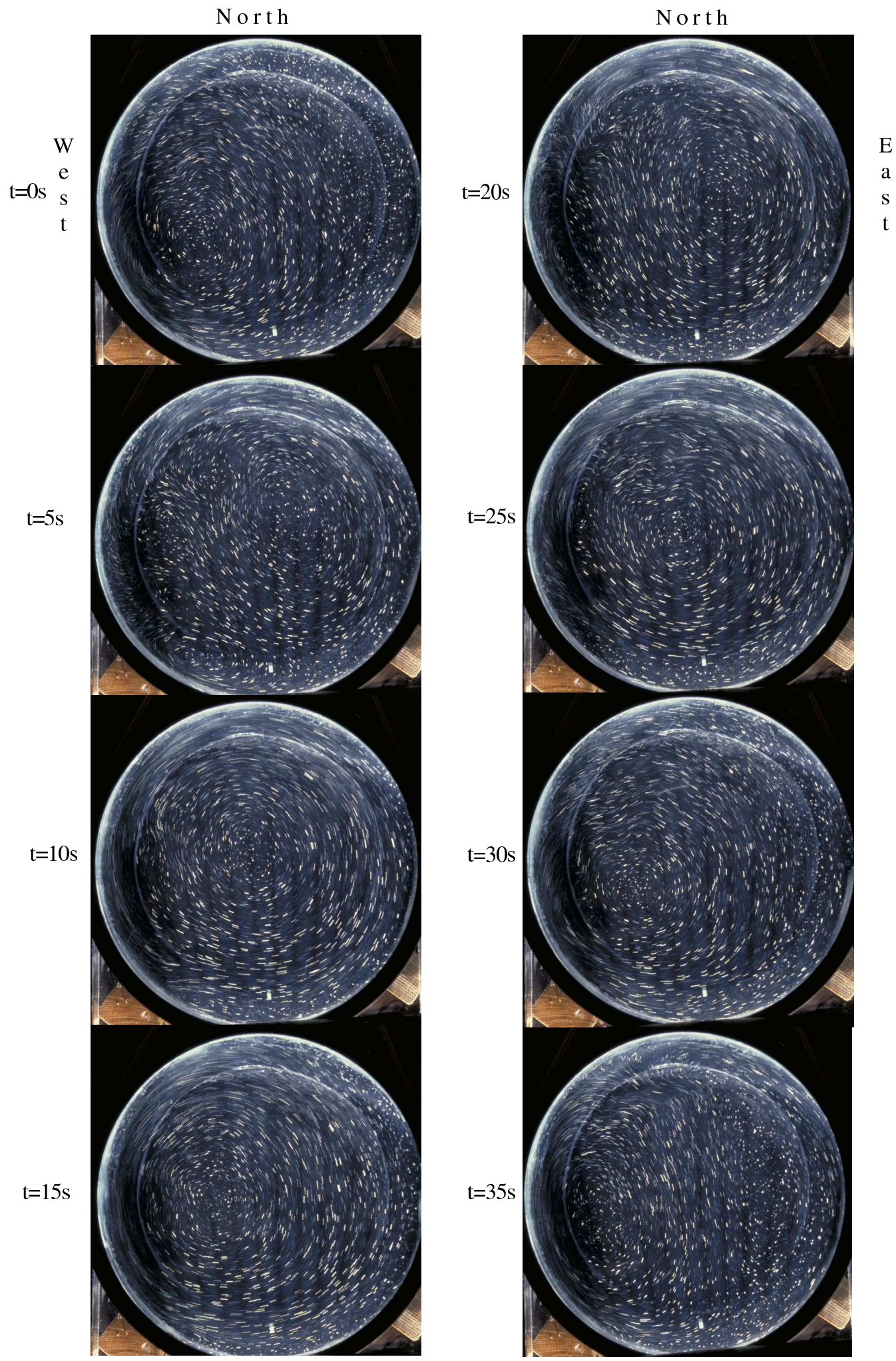

FIG. 13. Streak photography of hybrid-Rossby shelf modes at $t=0,5,10,15,20,25,30,35 \mathrm{~s}$ for a forcing period of 36.1s (nondimensional $\sigma=0.0871$ ), and maximum rigid-lid excursion $\Delta \theta=2 \pi$. 

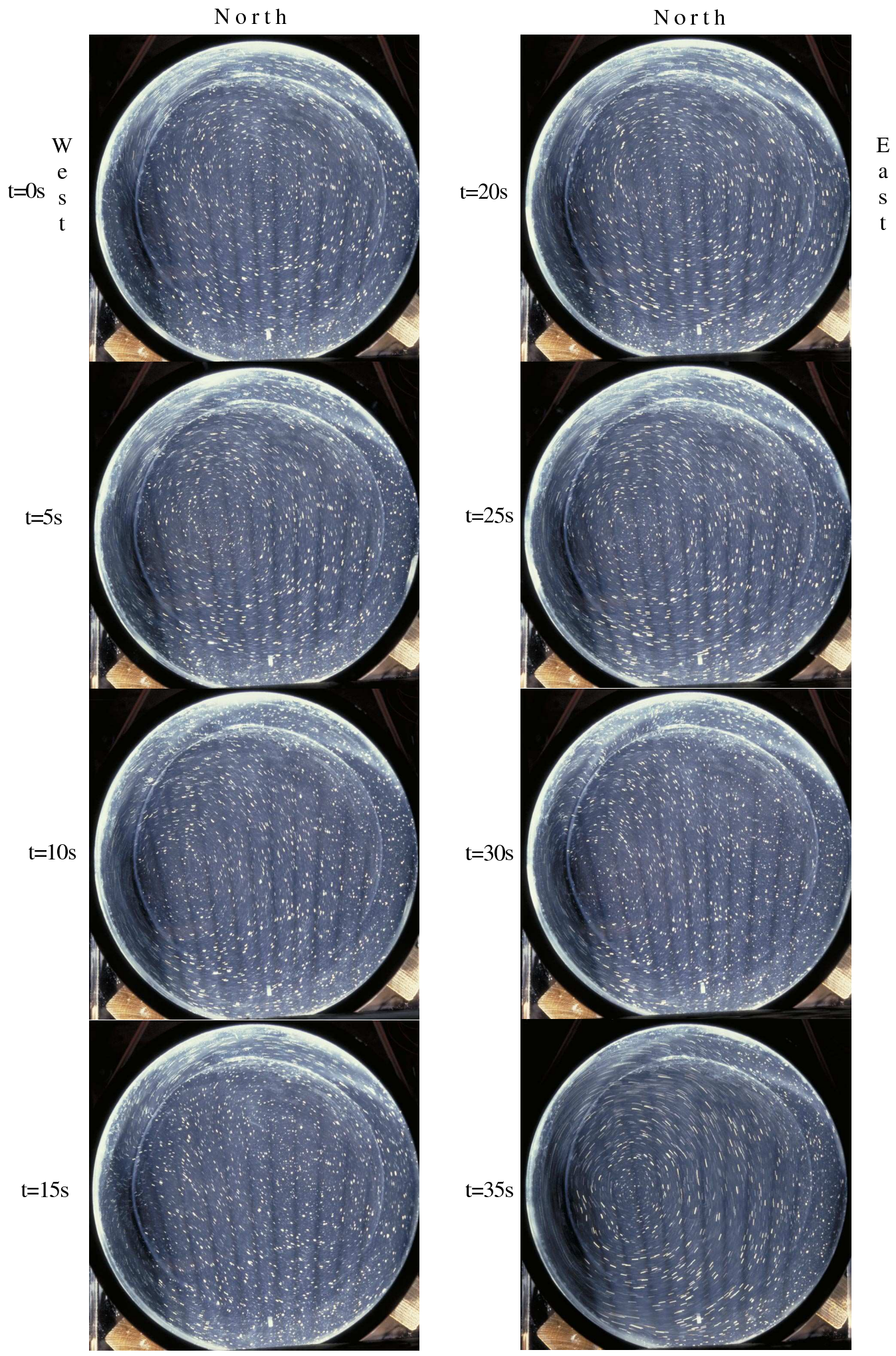

FIG. 14. Streak photography of hybrid-Rossby shelf modes at $t=0,5,10,15,20,25,30,35 \mathrm{~s}$ for a forcing period of 36.1s (nondimensional $\sigma=0.0871$ ), and maximum rigid-lid excursion $\Delta \theta=\pi / 2$. 


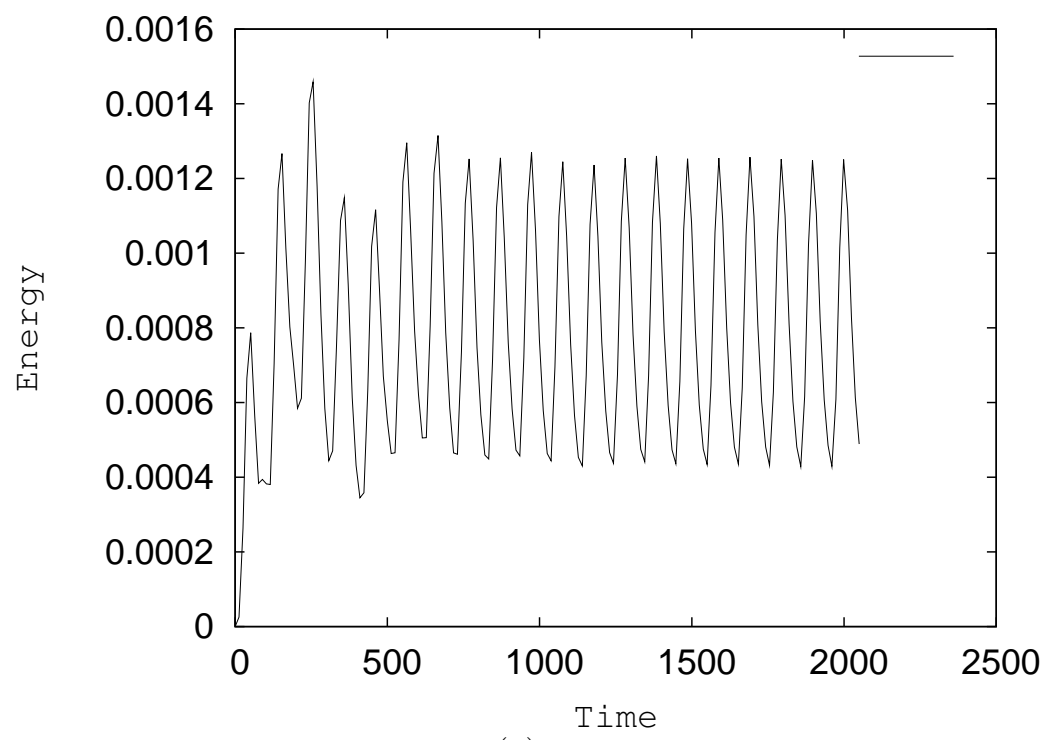

(a)

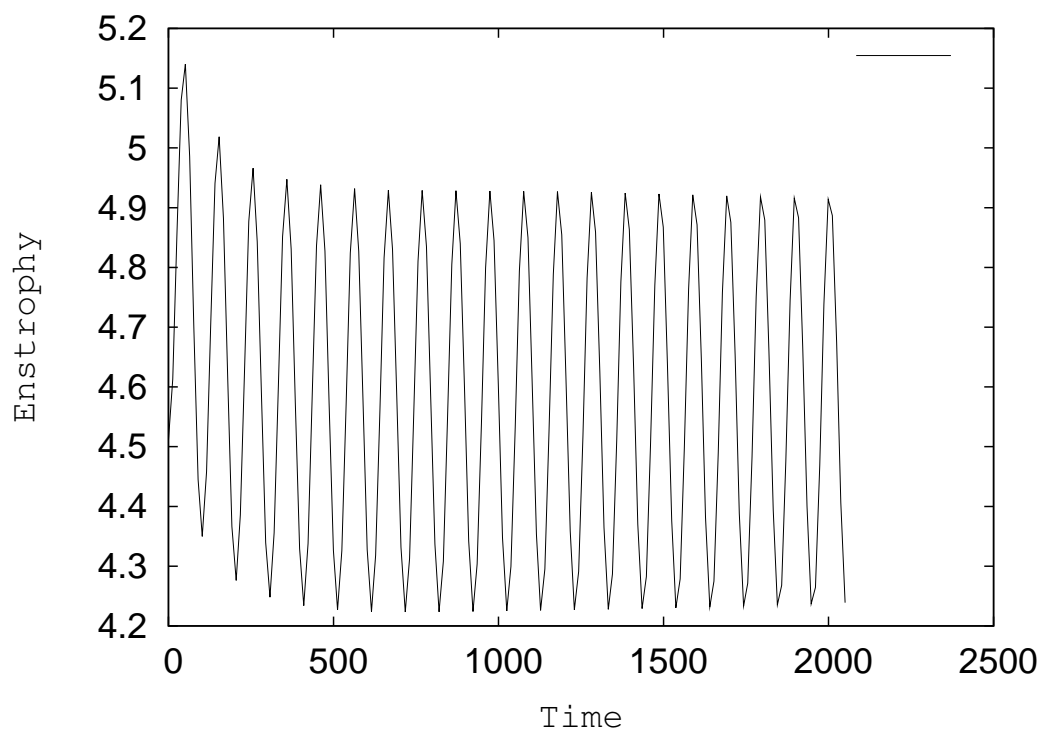

(b)

FIG. 15. Energy and enstrophy versus time. 

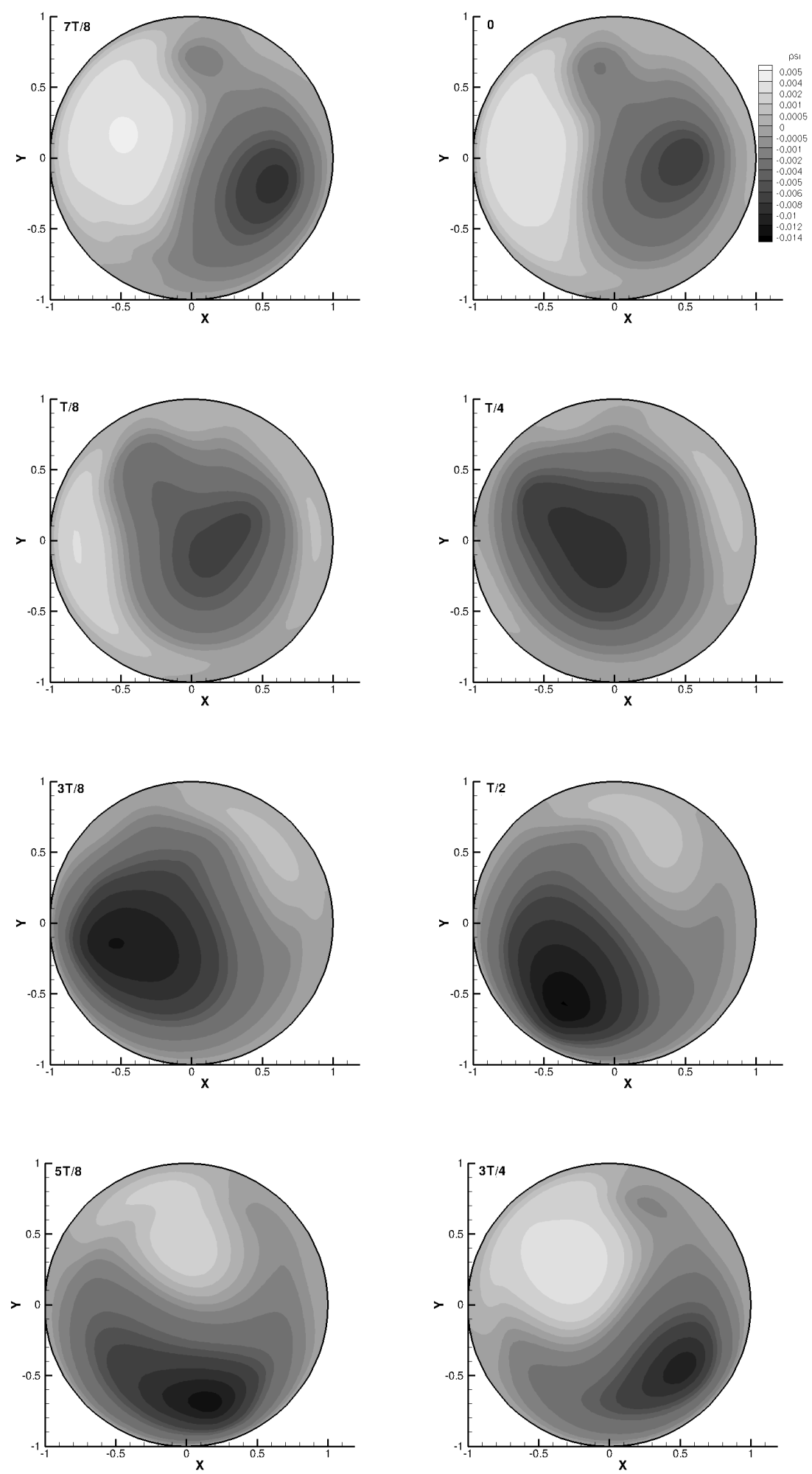

FIG. 16. Streamfunction over forcing period 20; $\sigma=0.0613, \kappa=0.0042, \epsilon=0.0314$, and $\Delta \theta=2 \pi$. 

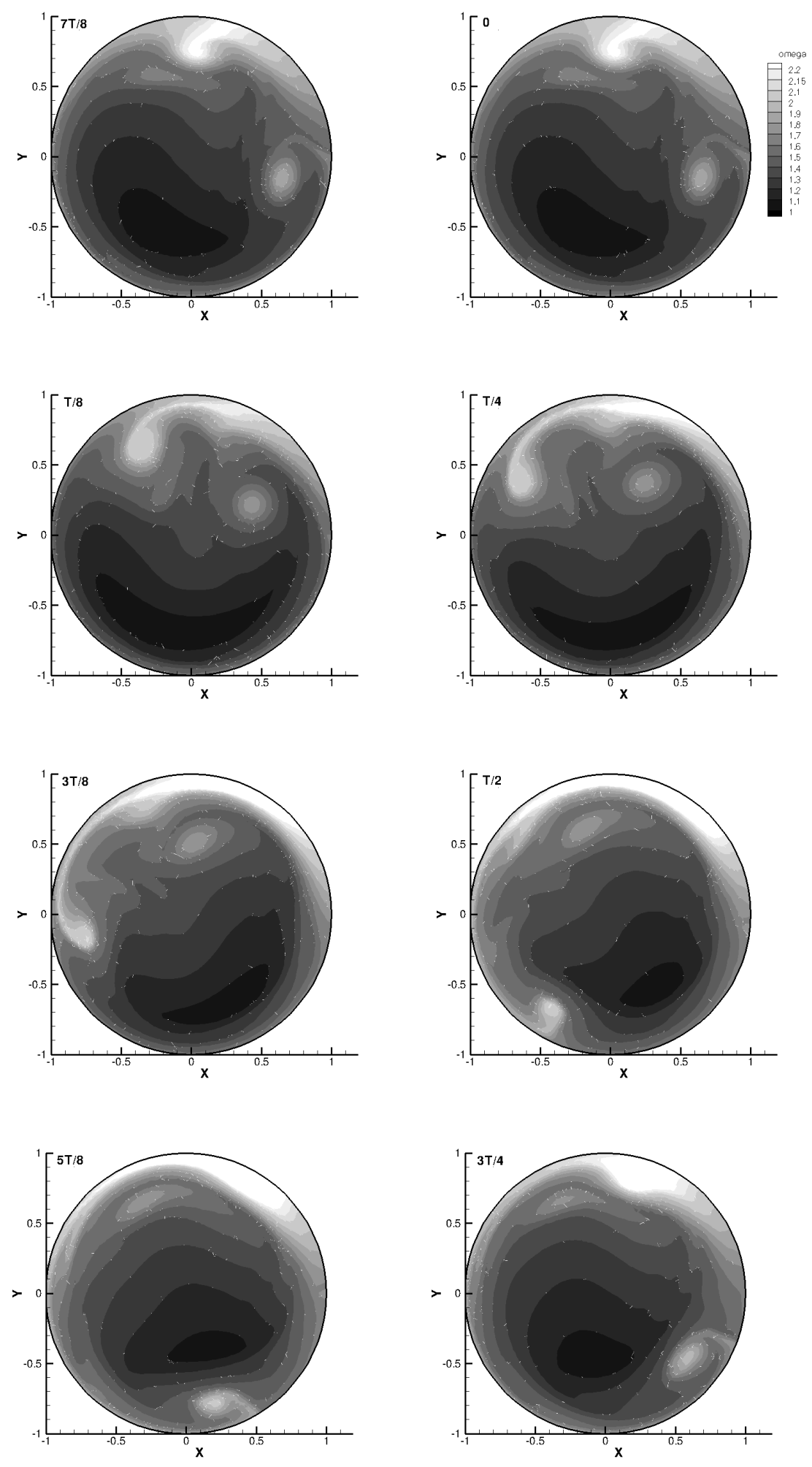

FIG. 17. Potential vorticity over forcing period 20; $\sigma=0.0613, \kappa=0.0042, \epsilon=0.0314$, and $\Delta \theta=2 \pi$. 

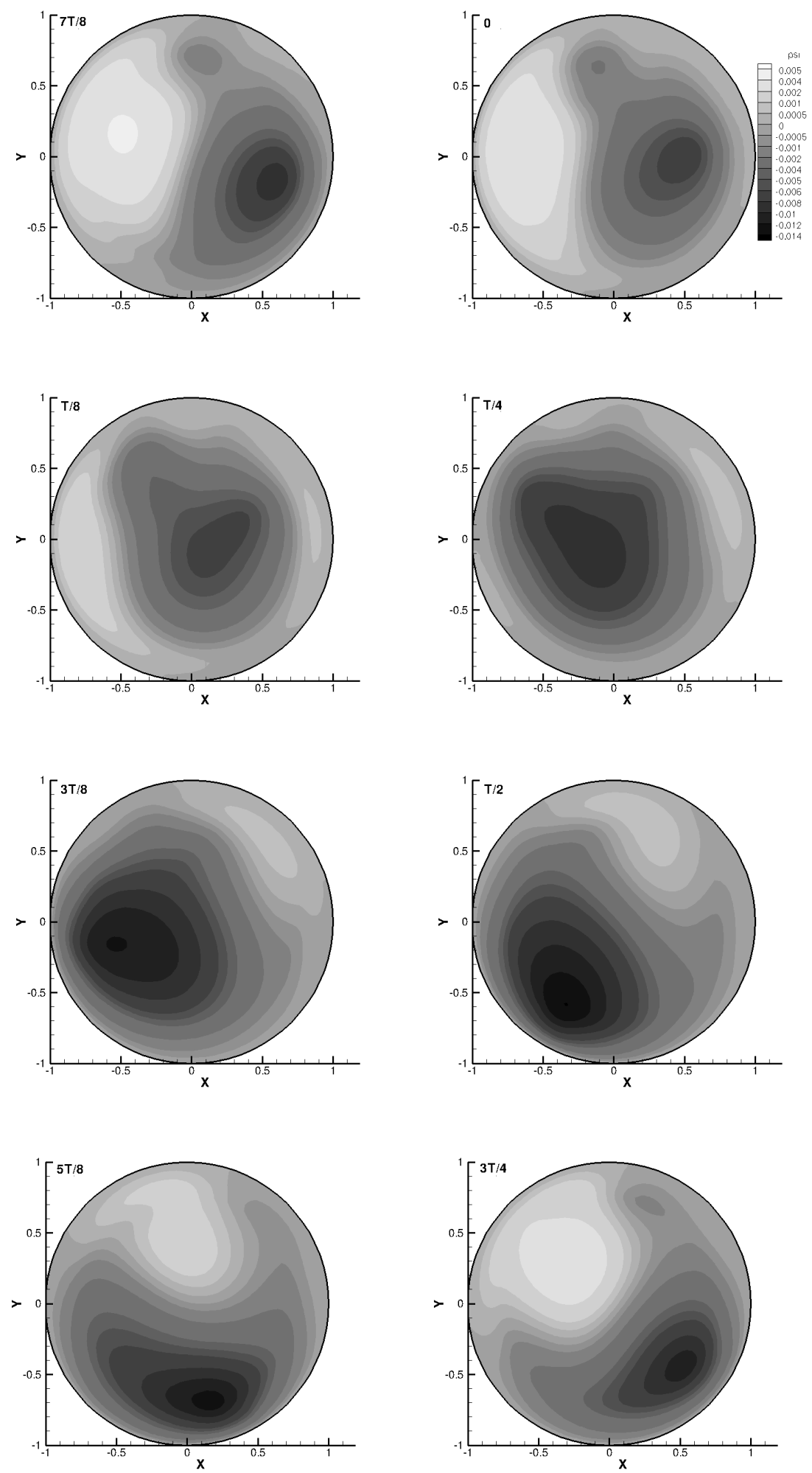

FIG. 18. Streamfunction fields over forcing period 19. 